\title{
L'ÉVOLUTION DU GENRE JAERA LEACH (ISOPODES; ASELLOTES) ET SES RAPPORTS AVEC L'HISTOIRE DE LA MÉDITERRANÉE
}

\author{
par \\ MICHEL VEUILLE \\ Laboratoire de Biologie et de Génétique Evolutives, C.N.R.S., 91190 Gif-sur-Yvette, France
}

\begin{abstract}
RESUME
Le genre Jaera comprend 19 espèces ou sous-espèces, pour la plupart européennes, dont les formes nouvelles Jaera istri, $J$. nordmanni nordmanni, J. n. occidentalis et J. n. illyrica. Il est possible de retracer leur phylogénie. Les événements cladogénétiques subis par ce groupe apparaissent étroitement corrélés à la formation des bassins méditerranéens et aux glaciations.
\end{abstract}

\section{ABSTRACT}

The genus Jaera includes 19 species or subspecies, mostly endemic to Europe. The new forms Jaera istri, J. nordmanni nordmanni, J. n. occidentalis, and $J . n$. illyrica are described. The phylogeny of this group can be inferred. The cladogenetic events leading to its differentiation appear closely correlated to the origin of the Mediterranean basin and to the glacial periods.

\section{INTRODUCTION}

Il est fréquent qu'un groupe dont la systématique est déjà établie nécessite une révision taxonomique par suite d'un intérêt accru qu'il suscite dans d'autres domaines de la recherche.

Il en va ainsi du genre Jaera Leach (1814). On pourrait le croire bien connu après les études de systématique descriptive réalisées il y a quelques dizaines d'années par Kesselyák (1938), Birstein (1951) et Karaman (1953). Mais, dès cette époque, ces organismes font l'objet d'investigations croissantes dans le domaine de la biologie des populations, qui remettent en cause ces travaux.

Forsman (1944, 1949) découvre chez Jaera albifrons des "races" différant entre elles par leur comportement sexuel, et que Bocquet (1953) élève au rang d'espèces jumelles, avant d'entreprendre l'étude génétique de leur spéciation, poursuivie depuis par Lécher (1967), Prunus (1968), Cléret (1970) et Cariou (1977).
D'autres auteurs élargissent ensuite au plan écologique l'étude de ce complexe spécifique (Naylor \& Haahtela, 1966, 1967; Naylor \& Slinn, 1958; Jażdżewski, 1969; Jones, 1972a \& b; Jones \& Naylor, 1971; Sjöberg, 1967, 1969, 1970; Steele \& Steele, 1972).

La reprise chez d'autres „espèces” de Kesselyák des travaux réalisés sur $J$. albifrons, met encore en évidence de nouveaux complexes spécifiques (Lemercier, 1960; Veuille, 1977; Veuille \& Kocatas, sous presse). Ces études ne se limitent pas à révéler dans un groupe animal une diversité insoupçonnée. Elles font surtout apparaître un foisonnement de formes à statut ,juxtaspécifique” (Bocquet, 1972), permettant de cerner au plus près le processus de spéciation et d'en démonter le mécanisme. Elles permettent entre autre, et c'est le but de ce travail, d'utiliser la localisation géographique souvent très stricte des espèces de Jaera pour mettre en relation leur radiation évolutive et les événements géologiques qui l'ont accompagnée, voire déterminée.

On suspecte en effet depuis G. O. Sars (1897) que l'évolution des Jaera est contemporaine de l'histoire de la Méditerranée. Dans cette perspective, les travaux de Valkanov (1938), Bacesco (1948) et Kothé (1968) ont prouvé que Jaera sarsi est une forme typique de la faune pontocaspienne.

Poursuivant cette idée, mes observations m'ont permis de constater que le genre Jaera dans son ensemble peut être subdivisé en différents groupes d'espèces apparentées ayant une origine géographique commune. Ces conclusions m'ont amené à reprendre l'étude synthétique du genre. Ce travail ne vise donc pas au premier chef la description d'espèces nouvelles ou l'étude détaillée de leur morphologie; il se limitera souvent à rappeler les données disséminées dans d'autres publications. 
Son objet est avant tout de repenser la systématique des Jaera en partant de bases nouvelles permettant d'en retracer l'histoire et de retrouver son association à la genèse des mers européennes.

\section{MATERIEL ET METHODES}

Depuis leur première mention par Leach (1814), les Jaera ont fait l'objet de nombreuses descriptions dont les critères se sont modifiés au fil du temps. L'étude des espèces jumelles a fait porter récemment la recherche des caractères discriminants au niveau des différenciations sexuelles externes, jusqu'alors négligées. C'est pourquoi je me suis basé en premier lieu sur l'observation de mes propres spécimens, pour ne faire appel aux publications d'autres auteurs que lorsque les figures fournies permettaient d'éliminer toute ambiguité quant au statut taxonomique réel des formes décrites. Il s'agit toujours de descriptions récentes. $\mathrm{Ce}$ tri amène à négliger de nombreuses localités citées dans la littérature, mais garantit l'exactitude des points retenus.

Une fois les espèces définies, la reconstruction de leur phylogénie pose un délicat problème de méthodologie. Plusieurs tentatives d'objectivation des ,caractères” empiriques de la systématique ont été proposées dans ce but, dont la taxonomie numérique (Sokal \& Sneath, 1963) et la méthode cladistique de Hennig (1966). Malheureusement, la taxonomie numérique ne permet pas de traiter les phénomènes de convergence, comme c'est le cas des espèces affines de $J$. sarsi et de $J$. massiliensis, qui possèdent en commun de nombreux caractères manifestement acquis de façon indépendante (voir ci-après).

L'autre défaut de la méthode numérique est d'attribuer le même poids à tous les caractères, ce qui entraîne une redondance lorsque ces caractères coévoluent, comme il advient généralement des caractères sexuels. Etant donné l'importance de ceux-ci dans la systématique des Jaera, on risquait de déséquilibrer fortement les distances calculées.

Quant à la méthode de Hennig, elle m'a semblé difficile à appliquer aux Jaera. Dans d'autres genres, ou à des niveaux taxonomiques plus élevés, il est souvent possible de définir sans ambiguité des caractères ",apomorphes" et „plésiomorphes". Mais l'évolution d'un caractère au sein d'un genre relève parfois, comme chez Jaera, d'un foisonnement et d'un éparpillement désordonné dans lequel il est malaisé de distinguer des types ancestraux et transformés.

J'ai préféré suivre une démarche méthodologique consistant à retrouver dans un premier temps les relations entre les espèces, sans a priori sur le sens de l'évolution des caractères. Le jeu de construction imaginaire consistant à représenter graphiquement les apparentements entre espèces perd alors le nom d',arbre", les figures horizontales ou verticales n'ayant plus de signification implicite.

La classification des espèces n'est alors possible qu'avec un effectif minimum de quatre: en effet, trois espèces ne peuvent être groupées que d'une seule manière, selon une figure en "Y" dont chaque taxon occuperait l'une des branches. Toutes les permutations sont équivalentes: que l'une des trois formes considérées soit morphologiquement différente des deux autres peut aussi bien signifier qu'elle est plus ,ancienne" que tenir au fait qu'elle a évolué plus vite, ou différemment, à partir de l'ancêtre commun ou de l'une des autres formes.

Ces ambiguités ne peuvent disparaître qu'à condition de disposer de quatre espèces. On peut classer celles-ci en les distribuant de façon imaginaire, soit aux différents sommets d'un „X", si aucune affinité n'est décelable, soit selon un „,H", si elles possèdent deux à deux des caractères alternatifs en commun: les apparentements suggérés par cette figure changent en effet selon les positions respectives attribuées à chacun des taxons. Cette méthode peut alors être poursuivie en intégrant progressivement d'autres espèces et en multipliant les branchements.

Cette procédure est plus générale que la classification cladistique, laquelle peut être dérivée de la précédente en disposant deux sémaphorons synapomorphes aux extrémités d'une même ,jambe" $\mathrm{du}$ "H”, les deux autres branches portant respectivement une espèce plésiomorphe, et l'enracinement du groupe étudié dans le reste du monde animal: l'hypothèse implicite est alors que l'espèce plésiomorphe possède en commun avec la condition ancestrale quelque caractère primitif alternatif de celui qui a permis de regrouper les deux 
formes les plus „évoluées”. La différence tient au fait que dans la présentation que l'utilise, aucun critère de "progrès" n'intervient, par suite d'une incertitude sur le sens d'évolution du caractère étudié: le temps n'est pas pris en compte au moment de l'analyse, mais seulement après et de manière facultative.

Un seul couple de caractères discriminants peut être retenu pour l'étude d'un événement cladogénétique, sous réserve que la biologie de ces caractères soit suffisamment connue pour permettre d'écarter les cas de convergence. C'est pourquoi ce travail s'est largement inspiré d'une étude antérieure portant sur la biologie de la reproduction chez Jaera (Veuille, 1978a \& b). La motivation originelle en est l'évolution considérable suivie par la sexualité des espèces de ce genre. Mais il faut remarquer en outre qu'aucune modification de cette fonction ne peut être versatile, par suite des contraintes sélectives qui convergent à ce niveau. La bonne connaissance théorique des phénomènes de sexualité, étudiés concurremment par plusieurs disciplines telles que l'écologie, l'éthologie et la physiologie, confère à leur étude une certaine transparence et une relative sûreté d'interprétation. Ce n'est pas le cas pour d'autres caractères discriminants couramment retenus par la systématique, tels que la chaetotaxie et les critères de forme, aux niveaux desquels les convergences évolutives sont fréquentes et imprévisibles.

\section{LE GENRE J $A E R A$}

Les Jaera sont des Isopodes Asellotes Parasellides de la famille des Janiridae. Leur taille varie de 1 à $5 \mathrm{~mm}$. Leur corps est très aplati du fait de sa compression dorso-ventrale et d'un pelstatisme variable mais parfois très développé qui distend sur les côtés des marges latérales du corps au point que les pleurépimères débordent l'insertion des péréiopodes. Pour cette raison, il n'y a pas de plaques coxales visibles en vue dorsale. Les marges latérales du pléotelson sont également étirées, sauf au niveau des uropodes, très courts, qui sont logés dorsalement dans une encoche unique de l'abdomen, non visible chez le jeune, mais de profondeur croissante avec la taille de l'individu ou de l'espèce: à peine distincte chez Jaera hopeana, elle est très profondément creusée chez les grands mâles de Jaera nordmanni.

Parmi les appendices caractéristiques du genre, il faut signaler les antennules, toujours très courtes, qui ne portent que de une à trois aesthétasques.

La morphologie des pièces buccales est d'un type généralisé (Lemercier, 1955; Veuille, 1977).

Les mandibules ont une „spine-row”, ainsi qu'une lacinia mobilis sur celle de gauche. Le palpe de trois articles porte deux soies sur le second.

La maxillule présente un précoxopodite élargi et un court coxopodite, avec quatre soies sur l'endite et douze phanères pectinés sur le basipodite. $\mathrm{La}$ maxille porte quatre soies sur chacun des lobes du basipodite. Les péréiopodes de la première paire portent deux ongles sur leur dactylopodite et leur coxopodite est fusionné au premier segment.

Les autres péréiopodes portent fondamentalement, sauf chez les trois espèces ponto-caspiennes, trois ongles sur le dactylopodite et un ergot triangulaire sur le propodite. Leur coxopodite est indépendant du thorax.

Les pléopodes respiratoires ont été décrits par Lemercier (1960). Leur principal caractère générique est l'absence totale des longues soies distales qui existent chez les autres Parasellides, à l'exception du genre Iais.

Les Iais semblent très proches des Jaera par leurs pléopodes respiratoires et leurs antennules courtes. Ils offrent en outre des ressemblances écologiques et comportementales frappantes avec J. hopeana. Ils se distinguent des Jaera par la présence de plaques coxales et l'absence d'encoche dorsale du pléotelson, ces deux caractères découlant de l'absence de pelstatisme.

On considèrera donc que les Jaera s'insèrent dans la famille des Janiridae au niveau des Iais. Mais au sein des Jaera eux-mêmes, différents groupes de parenté peuvent être mis en évidence.

Un premier groupe, que j'appellerai „Atlantique" rassemble les cinq formes de la super-espèce Jaera albifrons. Il s'agit de Jaera albifrons, Jaera ischiosetosa, Jaera posthirsuta, Jaera praehirsuta et Jaera forsmani.

Un second groupe, ,méditerranéen”, comprend les espèces Jaera italica, Jaera schellenbergi, Jaera nordmanni (comprenant plusieurs formes géogra- 
phiques), Jaera nordica (comprenant plusieurs formes géographiques), Jaera massiliensis et Jaera bocqueti.

Le groupe ponto-caspien sera constitué par Jaera sarsi, Jaera caspica et Jaera istri.

Le quatrième groupe comprend la seule espèce Jaera bopeana.

Cette classification évite de recourir aux catégories subgénériques habituellement employées dans la mesure où un tel groupe qui eût été qualifié de sous-genre par la systématique descriptive serait rangé comme super-espèce par la systématique évolutive (groupe atlantique), alors que tel autre „sous-genre" aurait compris simultanément en son sein des super-espèces et de „bonnes" espèces (groupe méditerranéen). Ces deux types de systématique emploient une nomenclature trinominale différente dont la confrontation eût apporté plus de confusion que de clarté, d'autant plus que le nom d'une sous-espèce de Jaera nordmanni eüt alors requis l'emploi d'une nomenclature pentanominale... A une précision excessive de la classification, j'ai donc préféré la mise en évidence des relations phylogéniques réelles.

Les quatre "groupes" présentés ci-dessus par anticipation seront définis tour à tour par leurs caractères sexuels et géographiques, qui permettent une comparaison membre à membre.

Mais il existe d'autres caractères qu'il est difficile de récapituler à chaque fois, soit qu'ils n'offrent de particularité notable que dans un petit nombre d'espèces, soit qu'ils gagnent à être comparés simultanément dans toutes les formes. Il faut cependant les mentionner, et pour éviter les descriptions fastidieuses et ne pas obliger le lecteur à de nombreux renvois, je les considèrerai ci-dessous globalement et de manière comparative:

- Les marges latérales du corps sont garnies d'épines alternativement longues et courtes chez $J$. hopeana, dans le groupe atlantique et dans le groupe méditerranéen, à l'exception de $J$. massiliensis et $J$. bocqueti. Ces deux dernières espèces et celles du groupe ponto-caspien offrent au contraire des phanères courtes, égales et densement serrées (figs. 5, 7, 8, 9).

- L'antennule porte trois aesthétasques chez $J$. bopeana, deux dans le groupe atlantique et une dans les groupes méditerranéen et ponto-caspien. - Les dactylopodites des pattes 2 à 7 portent deux ongles dans le groupe ponto-caspien et trois ongles dans les autres.

- Le maxillipède (fig. 1) possède un lobe élargi au niveau de ses trois articles proximaux, et un lobe étroit, dans les groupes atlantique et méditerranéen, à l'exception de $J$. bocqueti et $J$. massiliensis. Chez ces deux espèces et dans le groupe ponto-caspien, le palpe s'amincit et le lobe s'élargit corrélativement, pour former une lame couvrant les pièces buccales. Le maxillipède de $J$. bopeana est intermédiaire. Le lobe possède deux rangées de soies, sauf dans le groupe ponto-caspien, où la plus distale disparaît.

- Le rapport des tailles des articles distal et proximal de la troisième paire de pléopodes (fig. 2 ) décroît, quand on passe successivement de $J$. bopeana, aux espèces atlantiques, puis aux espèces méditerranéennes considérées dans l'ordre suivant: J. italica; J. schellenbergi; J. nordmanni et J. nordica; $J$. massiliensis et $J$. bocqueti. Cela est dû au fait qu'avec l'amincissement progressif de la taille des pléopodes 1 et 2 chez ces dernières espèces, le pléopode 3 acquiert peu à peu une fonction operculaire pour protéger la chambre respiratoire. L'exopodite acquiert alors une forme arrondie, sauf chez $J$. massiliensis et $J$. bocqueti où, comme dans le groupe ponto-caspien, il s'allonge en pointe pour coiffer une chambre respiratoire cordiforme.

\section{JAERA HOPEANA}

Jaera hopeana Costa, 1853

Jaera bopeana Costa, 1853.

Jaera kröyeri Rossi, 1906.

Jaera charrieri Monod, 1925.

Jaera hospitalis Verhoeff, 1943.

Jaera stagnoensis Verhoeff, 1949.

Jaera sulcata Verhoeff, 1949.

Jaera dalmatica Verhoeff, 1949.

Description. - A l'état adulte, Jaera hopeana mesure de 1 à $2 \mathrm{~mm}$, la femelle étant plus grande que le mâle. Les pleurépimères, peu développés, sont garnis de puissantes épines insérées sur une cuticule épaisse.

Comme dans toutes les espèces, les pléopodes 1 , première paire d'appendices copulateurs, sont fu- 
sionnés et constituent le préopercule (fig. 4a). Celui-ci est très élargi à sa base et s'amincit distalement pour former deux minces palettes arrondies au niveau desquelles naissent les cornes copulatrices, longues, recourbées, et pourvue de trois épines à leur apex.
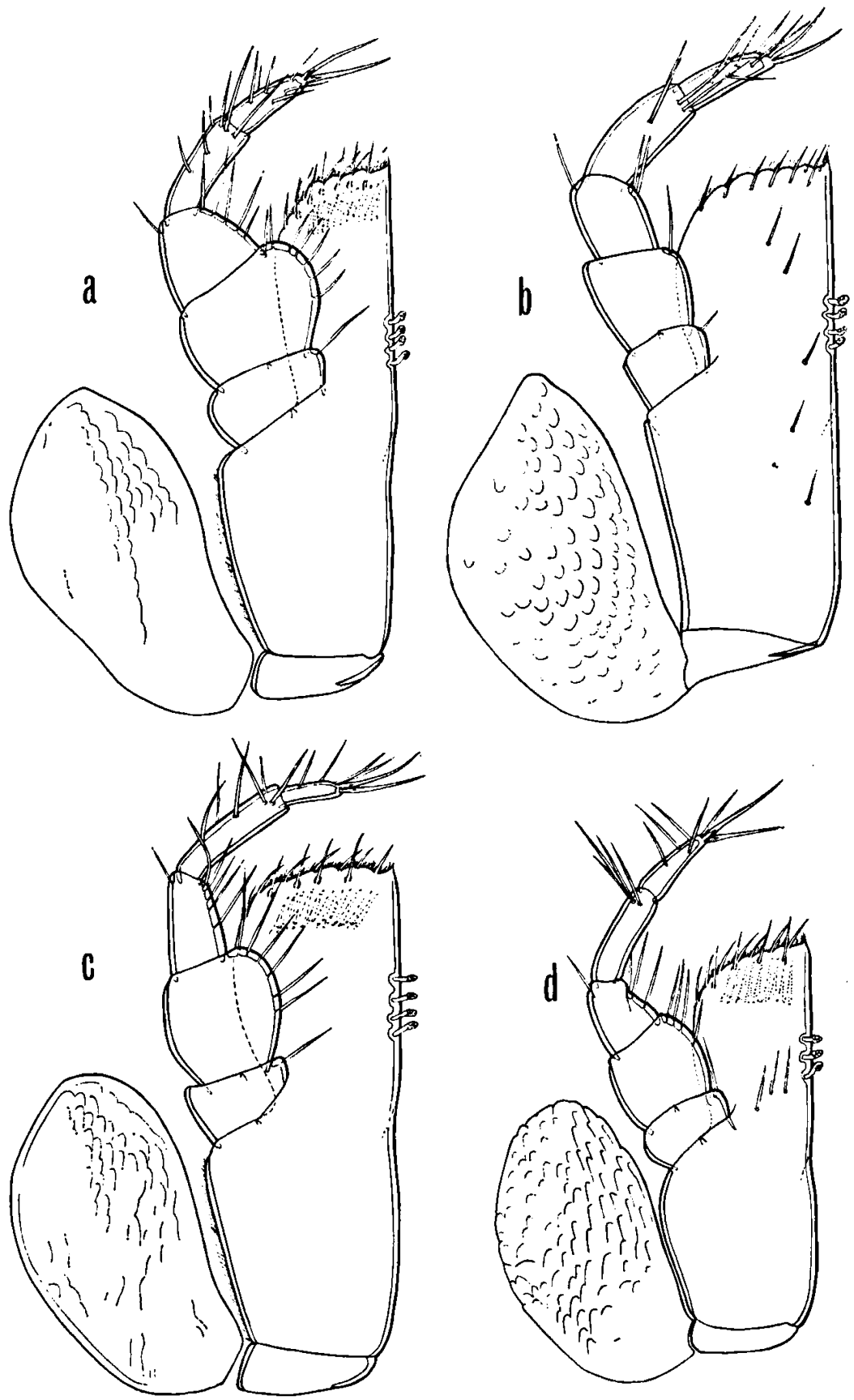

Fig. 1. Maxillipèdes: a, Jaera italica; b, J. istri; $c, J$. massiliensis; d, J. hopeana. 


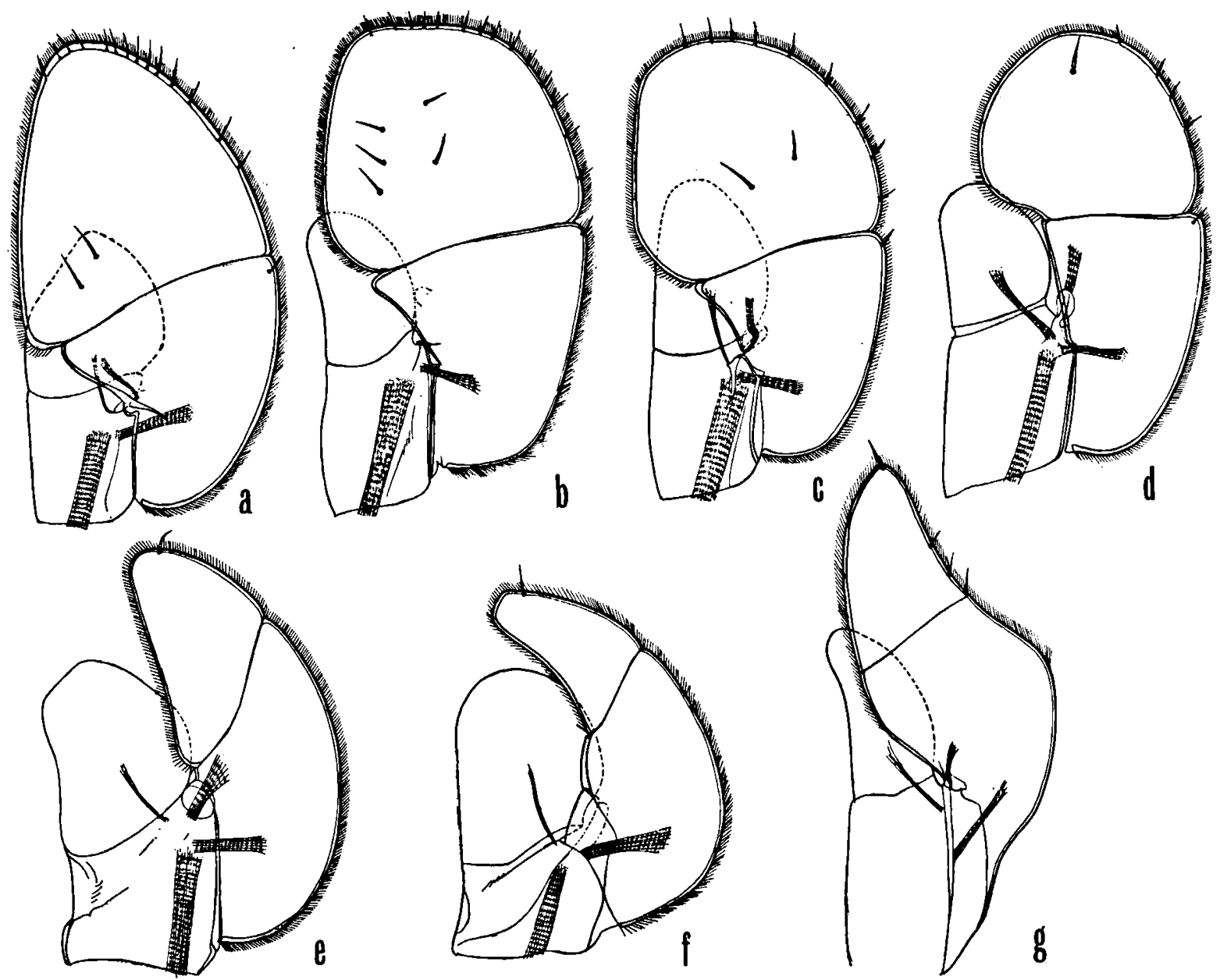

Fig. 2. Pléopodes 3 mâles: a, Jaera massiliensis; b, J. nordmanni occidentalis; c, J. schellenbergi; d, J. italica; e, J. albifrons; f, J. hopeana; $\mathrm{g}, J$. istri.

couverte d'expansions cuticulaires que forme l'exopodite fusionnée au sympodite du pléopode 2 .

L'orifice copulateur des femelles est constitué, comme chez tous les Jaera, d'une invagination de la cuticule à l'intérieur du corps, conduisant à la spermathèque (Veuille, 1978b). A chaque cycle sexuel, la femelle de $J$. hopeana synthétise un nouvel „organe cuticulaire” mais conserve les précédents qui se trouvent alignés en série à l'intérieur de l'organisme.

Les péréiopodes du mâle et de la femelle sont semblables, sauf au niveau du quatrième segment thoracique, dont les appendices sont transformés chez le mâle en crochets nuptiaux courts et trapus (fig. 3b). Les mâles utilisent parfois ces crochets pour se maintenir en position tête-bêche sur le dos de la femelle, mais il n'est pas encore possible d'affirmer qu'il s'agit d'un comportement copulateur. Ils tiennent les jeunes de stade I dans cette position à leur sortie du marsupium (fig. 3a). Ces néonates, qui sont indifféremment des deux sexes, se nourissent tandis que le mâle les maintient sur un Sphaerome: il pourrait s'agir d'un comportement paternel ou alloparental de soins aux jeunes mais sa parenté avec le comportement précopulateur des autres espèces est si frappante que l'on ne saurait exclure sa possible fonction reproductrice tant que la copulation n'aura pas été observé chez J. bopeana.

Les $J$. bopeana sont ectocommensaux des Isopodes Flabellifères de l'espèce Sphaeroma serratum (Fabricius, 1787), et vivent mêlés à leurs popula- 

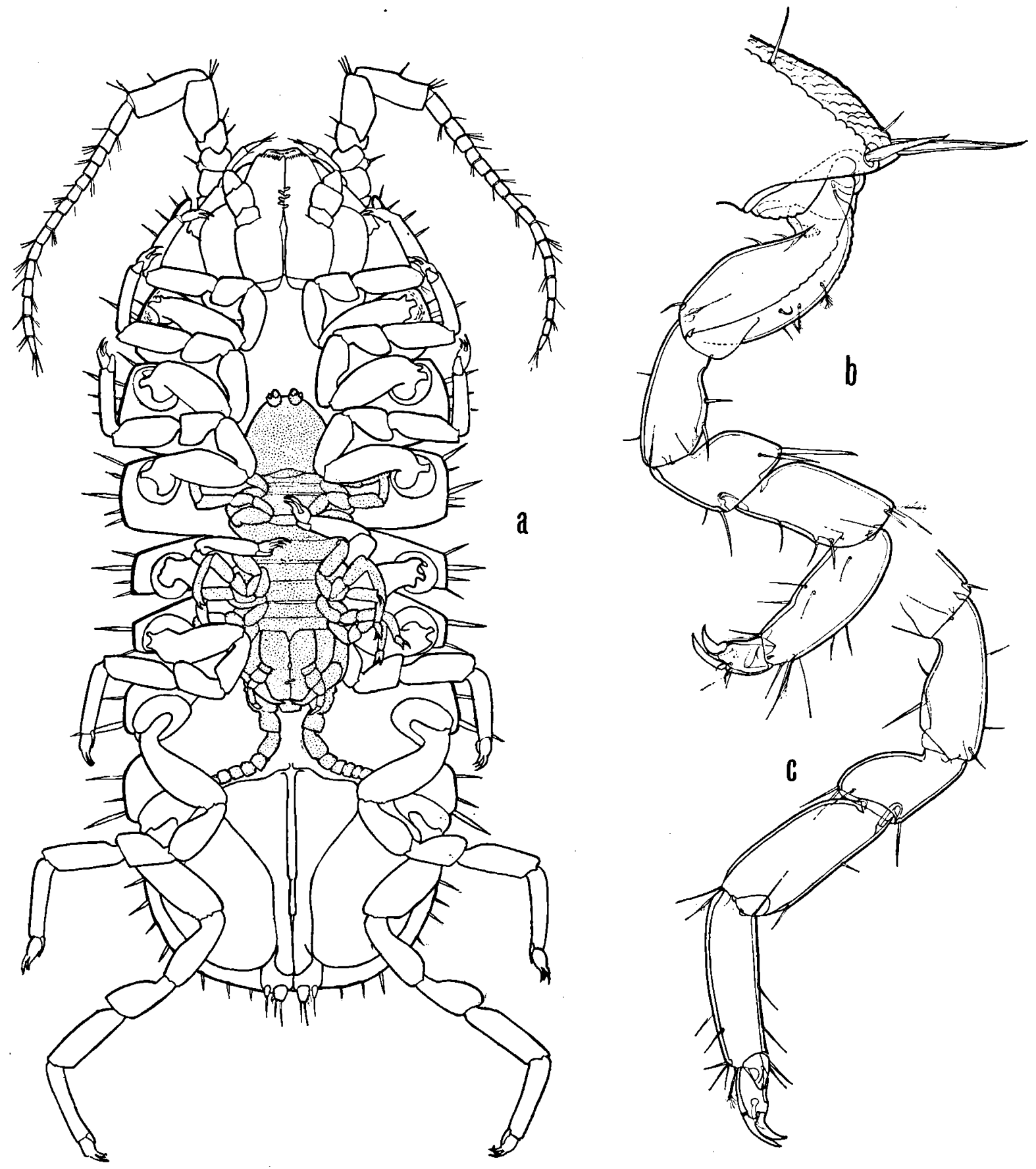

Fig. 3. a, Mâle de Jaera bopeana maintenant un jeune de stade 1 en position tête-bêche à l'aide des péréiopodes 4; b, péréiopode 4 mâle de $J$. bopeana; c, péréiopode 4 femelle de $J$. bopeana. 
tions, tantôt sous les mêmes rochers littoraux, tantôt sur les Sphaeromes eux-mêmes. En élevage, ils paraissent se nourrir de la faune fixée à la cuticule de ceux-ci.

Distribution géographique.- On trouve J. hopeana dans tous les bassins méditerranéens: Mer Egée (Veuille \& Kocatas, sous presse), Méditerranée occidentale, Adriatique (observations personnelles), Afrique du Nord (Prunus \& Pantoustier, 1976). Monod (1925) la signale sur les côtes marocaines de l'Atlantique, et Naylor (1972) dans la Manche.

Remarques phylogéniques. - Malgré son appartenance incontestable au genre Jaera, J. hopeana diffère beaucoup des autres espèces par sa biologie et sa morphologie. Elle possède en commun avec le groupe atlantique le faible pelstatisme, le dimorphisme de la taille en faveur des femelles et la conservation par la femelle, d'un cycle sexuel sur l'autre, des anciens organes cuticulaires et des produits génitaux reçus dans sa spermathèque (Veuille, 1978b).

Elle a de nombreuses affinités avec le genre Iais, également ectocommensal des Sphaeromides (Menzies \& Barnard, 1951; Hurley, 1956; Rotramel, 1975), qui possède le même comportement ,paternel" (Coineau, 1971). La présence de ce genre dans l'Antarctique et l'Indo-pacifique contraste avec la distribution méditerranéenne de $J$. hopeana dont l'isolement date sans doute de l'individualisation de la Méditerranée.

\section{LE GROUPE ATLANTIQUE}

Description. - Cet ensemble est constitué par la super-espèce Jaera albifrons, très étudiée, dont je rappellerai ci-dessous les caractères principaux:

La femelle, longue de $5 \mathrm{~mm}$ en moyenne, est presque deux fois plus grande que le mâle. Leur pelstatisme est médiocre. Le préopercule des mâles montre sa plus grande largeur à son extrémité distale et affecte la forme d'un „T"' (fig. 4d); il porte à ce niveau des cornes assez courtes dirigées antérieurement et deux mucrons proéminents à ses angles distaux externes.
Les pléopodes 2 se signalent par un exopodite en forme de lamelle arrondie détachée du sympodite (fig. 4c). Les deux paires d'appendices copulateurs sont identiques dans les différentes espèces. Elles sont coaptées pour former au repos un opercule recouvrant les pléopodes respiratoires.

L'organe copulateur des femelles est également identique entre les espèces. C'est un ensemble pluristratifié constitué par la rétention, d'un cycle sexuel sur l'autre, d'organes cuticulaires empilés en „oignon" (Veuille, 1978b).

Ce groupe compte cinq espèces interfécondes dont les femelles sont semblables, mais dont les mâles diffèrent par les peignes sexuels. Ceux-ci sont constitués par des soies que portent les péréiopodes des trois paires antérieures, chez $J$. praebirsuta et $J$. forsmani, ou les paires 6 et 7 , chez $J$. iscbiosetosa, J. albifrons et $J$. posthirsuta. Ces espèces se distinguent alors par les articles sur lesquels s'implantent ces formations (voir la monographie de Bocquet, 1953). Les paires 4 et 5 sont sexuellement indifférenciées, mais permettent au mâle de se maintenir en position "tête-bêche" sur le dos de la femelle lors de la parade sexuelle.

On distingue:

Jaera albifrons Leach, 1814

= Jaera albifrons sensu Bocquet, 1953; Jaera albifrons syei Bocquet, 1953. Cette espèce a dans l'Atlantique nord une distribution septentrionale qui va de la frontière franco-espagnole à la Nouvelle Angleterre, incluant la Baltique, la Mer Blanche et le Groënland.

Jaera ischiosetosa Forsman, 1949

Ayant la même distribution.

Jaera praehirsuta Forsman, 1949

Ayant la même distribution.

\section{Jaera posthirsuta Forsman, 1949}

Cette espèce est exclusivement nord-américaine. Les populations sont situées d'une part sur la côte est des Etats-Unis, au sud de Boston, et d'autre part dans les refuges thermiques à faune relicte post-glaciaire de la côte est du Canada (Veuille, 1976). 

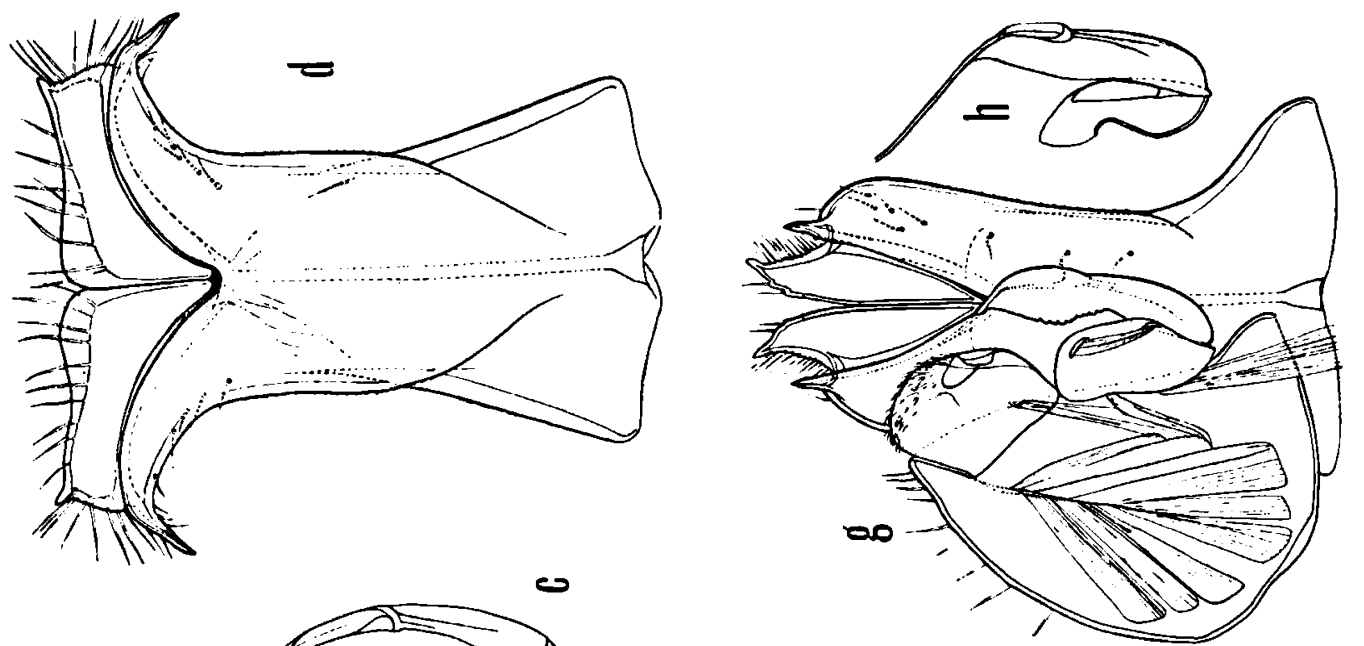

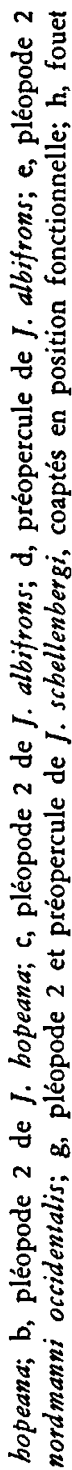
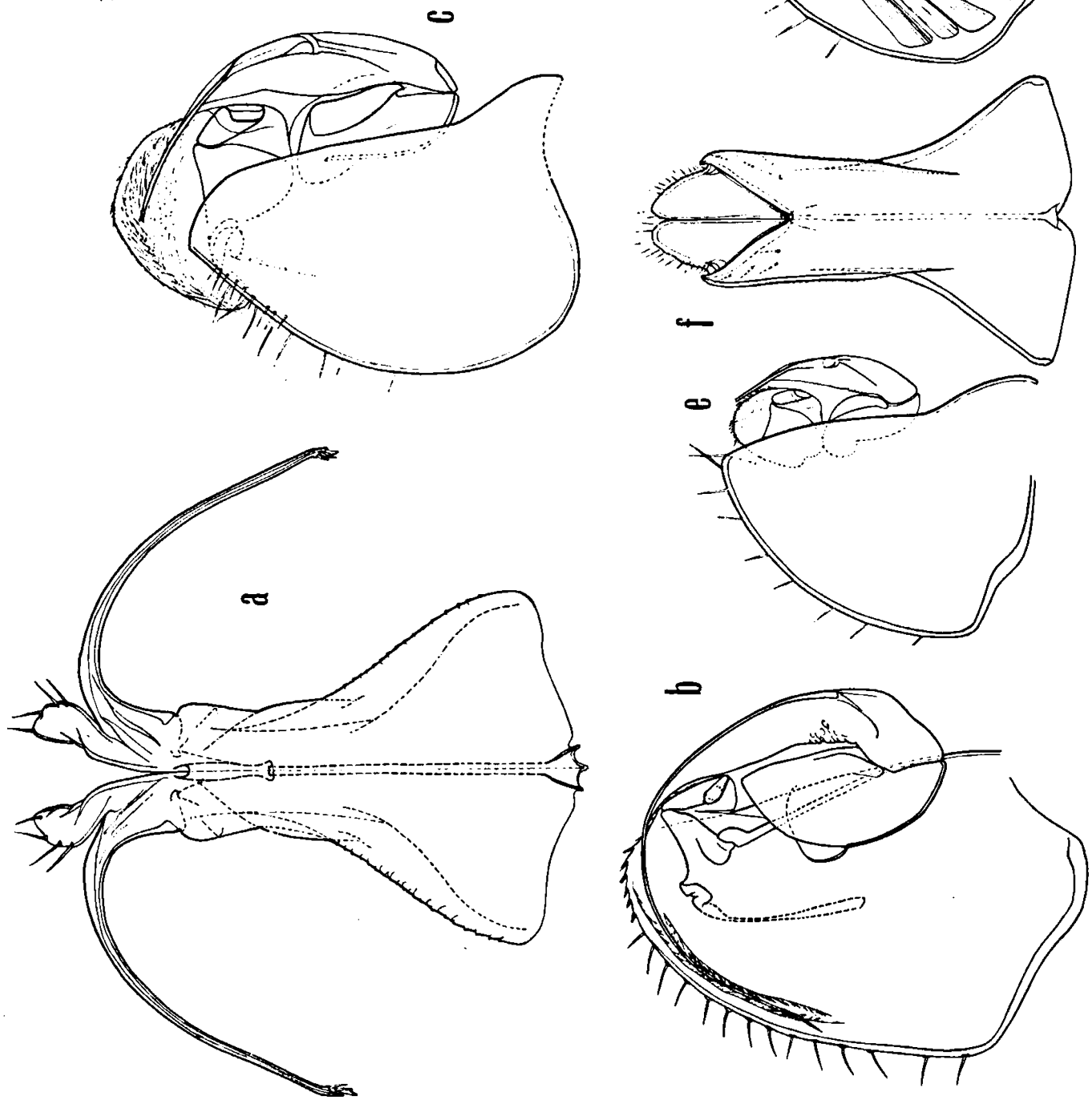

范

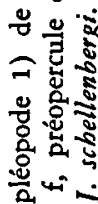

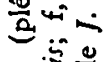

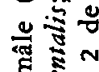

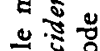

苞客

응

造

तो है

活范范 
Jaera forsmani Bocquet, 1953

Espèce endémique de la Manche et la Mer d'Irlande (Naylor, 1972).

Remarques phylogéniques. - Les cinq espèces sont très proches les unes des autres et il est difficile d'en retrouver la phylogénie. Il est tentant de les diviser selon que les variants sexuels se situent sur les péréiopodes postérieurs ou sur les pattes antérieures, cette dernière disposition rappelant les différenciations sexuelles des mâles du groupe méditerranéen résume sans doute l'état ancestral. Par l'étude des distances génétiques, Cariou (1977) établit deux groupes d'affinités, l'un constitué de $J$. praebirsuta et $J$. forsmani, l'autre étant formé de J. ischiosetosa et J. albifrons.

\section{LE GROUPE MEDITERRANEEN}

Description. - A l'inverse du groupe atlantique, les espèces méditerranéennes se reconnaissent entre elles par les caractères sexuels primaires des mâles. Les variants sexuels se rapportent au contraire au même type de base, caractéristique de tout le groupe. Ils ne subissent de variations que dans les cas peu nombreux où des espèces ou leurs sous-espèces se rencontrent en condition sympatrique.

Le pelstatisme est accusé, surtout chez le mâle, qui est à la fois plus long et plus large que la femelle.

Les pléopodes 1 varient d'une espèce à l'autre selon une évolution qui voit les cornes du préopercule changer d'orientation. Encore longues et recourbées en "T"' chez Jaera italica, elles se raccourcissent et se redressent postérieurement chez les autres espèces pour former un préopercule en forme de "Y".

Les pléopodes 2 possèdent un exopodite lamelleux arrondi, et un fouet copulateur de longueur variable corrélée à celle des cornes du préopercule dans lesquelles elles coulissent lors de l'insémination.

Ces deux paires d'appendices diminuent simultanément de taille et perdent leur fonction operculaire au profit des pléopodes 3.

L'orifice copulateur des femelles est un organe unique, rejeté à chaque cycle sexuel avec l'exuvie du stade sans oostégites. Sa profondeur est fonction de la longueur des cornes copulatrices du mâle de même espèce (Veuille, 1978b).

Les trois paires de pattes antérieures portent de longues soies constituant des peignes sexuels sur le propodite, carpopodite, méropodite, ischiopodite (Kesselyák, 1938), sauf chez J. bocqueti (Veuille \& Kocatas, sous presse), dont les appendices correspondants sont glabres (c'est à tort que Kesselyák mentionne aussi leur absence chez J. schellenbergi). Chez de grands individus de certaines espèces, les mêmes différenciations apparaissent aussi chez les femelles ou sur d'autres péréiopodes des mâles, mais leur usage systématique est limité.

Les péréiopodes de la quatrième paire sont courts et transformés en crochets nuptiaux armés de plusieurs rangées d'épines sur le carpopodite. Ils se referment comme une pince pour tenir la femelle pendant la copulation. Celle-ci se fait, comme chez les autres Jaera, en position tête-bêche, le mâle tenant sous lui le dos de la femelle. Pendant la précopulation, il tourne vers lui la face ventrale de la femelle, la tête étant orientée vers l'avant du mâle, en utilisant sa taille supérieure pour la maintenir entre ses pattes au moyen des péréiopodes 4 (fig. 5a).

\section{Jaera italica Kesselyák, 1938}

On reconnaît cette espèce aux pléopodes copulateurs en forme de „T' du mâle (Veuille, 1978a) et au long article proximal de l'exopodite des pléopodes 3 (fig. 2d). J. italica vit en milieu saumâtre, dans les lacs côtiers ou au débouché des rivières. Sa répartition géographique se limite à la partie orientale de la Méditerranée, avec une faible pénétration dans la partie méridionale des autres bassins. Elle comprend la Mer du Levant et le sud de la Mer Egée (Veuille \& Kocatas, sous presse), la Crête (Schmalfuss, 1975), la Grèce (Strouhal, 1942), le sud de l'Adriatique (observations personnelles; Kesselyák, 1938; Karaman, 1953; Sket, 1969), et le Cap Bon, à la pointe nord-est de la Tunisie (Prunus \& Pantoustier, 1976). Elle est absente de la Méditerranée occidentale et des régions nord de l'Adriatique et de la Mer Egée. 
Jaera schellenbergi Kesselyák, 1938

Cette forme se distingue des espèces dont le pléopode 1 est redressé en direction caudale par ses mucrons et ses cornes copulatrices relativement longs, et par l'élargissement subdistal des préopercules, qui simule encore la forme évasée que l'on trouve chez les espèces à organe copulateur en „T" (fig. 4g).

J. schellenbergi habite les lacs saumâtres et les sources karstiques littorales du Kvarner et du Golfe de Venise, à l'extrémité septentrionale de l'Adriatique (Kesselyák, 1938; Karaman, 1953; Sket, 1969). C'est une région aux hivers rudes et au climat plus froid que celle que colonise $J$. italica. Les deux espèces, l'une au nord, l'autre au sud, s'excluent mutuellement de part et d'autre d'une frontière passant par le Lac de Proklian, près de Chibenik (Yougoslavie), bras de mer aux eaux saumâtres où elles cohabitent dans des stations différentes.

Jaera nordmanni (Rathke, 1837)

= Jaera (nordmanni) nordmanni; Lemercier, 1960. Selon la description de Lemercier, cette espèce se reconnaît à son préopercule aux bords subparallèles se terminant par deux mucrons pointus, et flanqué de deux petites cornes subdistales orientées caudalement. Cette forme typique ne se rencontre que dans les populations de la Méditerranée occidentale.
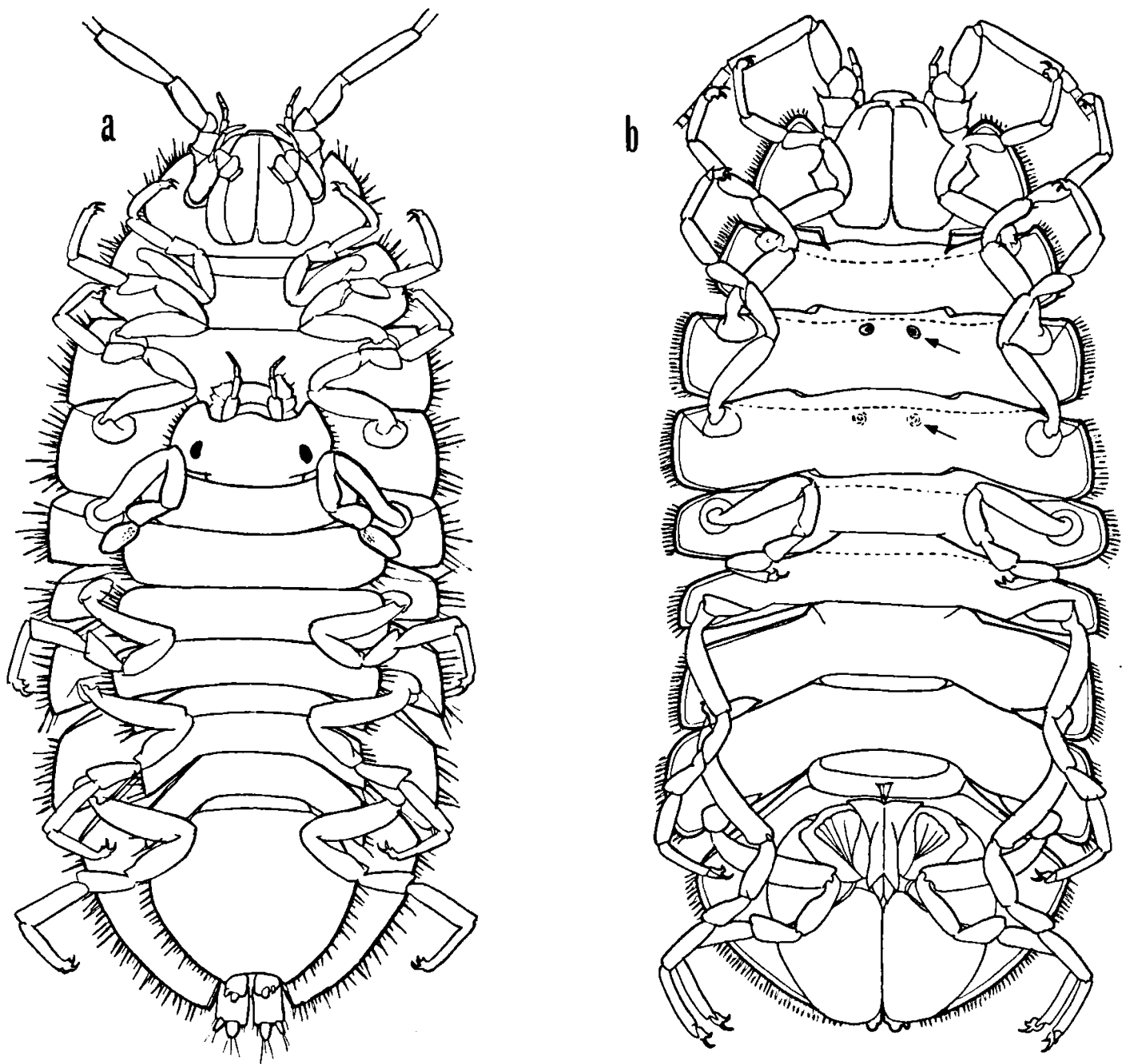

Fig. 5. a, Couple de Jaera italica en préparation à la copulation: le mâle maintient la femelle au moyen des péréiopodes 4 (vue ventrale); b, mâle de $J$. bocqueti en vue ventrale montrant les protubérances des segments 2 et 3 (flèches). 
Les mucrons manquent chez les mâles des trois stations prospectées sur la côte Dalmate (fig. 4f) et leurs cornes sont courtes. Les exemplaires turcs décrits par Veuille \& Kocatas (sous presse) diffèrent quant à eux par leurs mucrons de taille médiocre et leurs cornes très recourbées vers l'axe du corps.

Enfin, les populations des Açores montrent des cornes recourbées vers la marge du préopercule (Veuille, 1977) avec des mucrons très détachés. Les populations d'une même province sont semblables, sans intergradations entre elles, par suite de l'absence des populations intermédiaires pouvant faire la jonction d'un bassin à l'autre. L'espèce est donc scindée en quatre groupes morphologiquement caractérisables dont il convient de faire les quatre sous-espèces J. nordmanni nordmanni (Mer Egée et Mer Noire), J. nordmanni illyrica n. ssp. (Adriatique), J. nordmanni occidentalis n. ssp. (Méditerranée occidentale) et $J$. nordmanni guernei Veuille, 1977 (Açores).

Les exemplaires turcs on été récoltés dans la Mer Noire et la Mer Egée, au sud de la péninsule d'Izmir. Ceux de l'Adriatique proviennent d'une étroite bande côtière des environs de Split, allant de Jadrtovac à Drvenik (Yougoslavie), soit dans la partie nord de la distribution de I. italica. Les J. nordmanni occidentalis se situent à l'ouest d'une limite approximative passant par Tunis. Les stations saumâtres de Jaera situées plus au sud abritent invariablement $J$. italica. Il semble que cette espèce limite ainsi l'extension méridionale des $J$. nordmanni. dont l'aire de répartition se trouve fragmentée puisque les bassins de la Méditerranée communiquent entre eux par le sud: l'absence de migrations qui en résulte rend compte du début de différenciation morphologique des quatre sousespèces. I. nordmanni est une espèce d'eau saumâtre comme $J$. italica et $J$. schellenbergi. Elle s'observe en sympatrie avec $J$. italica dans la seule localité de Drvenik (Dalmatie), au débouché marin d'un ruisseau où les deux populations vivent côte à côte, les J. nordmanni en amont des J. italica. La forme guernei colonise les rivières de l'archipel des Açores, de la côte à une altitude de $500 \mathrm{~m}$.

Le type de $J . n$. illyrica est un mâle de $1,72 \mathrm{~mm}$, originaire de Jadrtovac (Yougoslavie) et déposé au Muséum National d'Histoire Naturelle, Paris.
Le type de $J . n$. occidentalis est un mâle de $1,72 \mathrm{~mm}$, originaire de Calvi (Corse), déposé au Muséum National d'Histoire Naturelle, Paris.

Jaera nordica Lemercier, 1958

= Jaera (nordmanni) nordica; Lemercier, 1960. Dans la description de Lemercier cette espèce se distingue des $J$. nordmanni par l'implantation perpendiculaire des cornes copulatrices sur le préopercule. Les mucrons manquent dans la sous-espèce J. nordica insulana Veuille, 1977.

J. nordica nordica est connue des eaux saumâtres supralittorales de la Manche et de la côte basque. J. nordica insulana vit dans les rivières d'eau douce des Açores.

Jaera massiliensis Lemercier, 1958

= Jaera (nordmanni) massiliensis; Lemercier, 1960. Le préopercule de cette espèce est assez semblable à celui de J. nordica (Lemercier, 1960), mais les deux espèces se distinguent facilement par leur morphologie. Les épines courtes et égales de la marge latérale du corps, le maxillipède au lobe élargi (fig. 1c), le pléotelson cordiforme de la femelle et les pléopodes 3 allongés (fig. 2a), caractères qui rappelleront ceux des espèces pontocaspiennes, les différencient des espèces $J$. nordmanni et $J$. nordica, pourtant très proches.

Le mâle possède en outre des tubercules externes en relief sur la face ventrale des 2ème et 3ème segments thoraciques (fig. 5b). Ces protubérances creuses qui manquent chez les espèces précédentes sont placées au niveau où se loge la tête de la femelle pendant la précopulation, et jouent sans doute un rôle lors de celle-ci.

J. massiliensis est une espèce littorale marine colonisant tous les bassins méditerranéens (Mer Egée, Adriatique, Méditerranée centrale et occidentale). Lemercier (1968), la signale à Madère.

Jaera bocqueti Veuille \& Kocatas n. sp.

Cette espèce est jumelle de $J$. massiliensis et ne s'en distingue que par l'absence de soies sur les trois paires antérieures de péréiopodes et l'orientation caudale des cornes des préopercules (Veuille \& Kocatas, sous presse). Elle est connue d'une station littorale de l'île de Bozcaada, au sud du détroit des Dardanelles (Mer Egée). 
Le type est un mâle de $2,78 \mathrm{~mm}$, déposé au Muséum National d'Histoire Naturelle, Paris.

Remarques phylogéniques. - Les espèces méditerranéennes de Jaera forment un groupe très diversifié, où $J$. italica constitue une forme charnière avec les autres groupes d'une part, et avec J. schellenbergi de l'autre. Les espèces restantes forment un sous-ensemble très homogène dont le préopercule, malgré ses variations, reste très semblable. Lemercier les a rassemblées en une super-espèce ,Jaera nordmanni", incluant J. (nordmanni) nordmanni, $J$. (nordmanni) nordica et $J$. (nordmanni) massiliensis. Cette classification doit être abandonnée puisque chaque membre de cette super-espèce constitue un complexe spécifique à part entière: $J$. massiliensis s'avère posséder une espèce jumelle $J$. bocqueti; quant à $J$. nordmanni et $J$. nordica, elles connaissent une division en sous-espèces, dont au moins les formes des Açores possèdent suffisamment de caractéristiques morphologiques (voir Veuille, 1977) pour faire suspecter un degré élevé de différenciation évolutive qui ne pourra être étudié que par des techniques génétiques.

\section{LE GROUPE PONTO-CASPIEN}

D e s cription. - Dans les trois espèces de ce groupe, les deux sexes sont de longueur semblable et montrent un pelstatisme accusé.

Le préopercule, étroit à sa base, s'évase vers le sommet pour donner par une courbure régulière deux cornes qui se déploient vers l'extérieur (fig. $6 \mathrm{c}-\mathrm{e})$. La bordure distale de l'organe montre deux lames semi-circulaires dépourvues de mucrons.
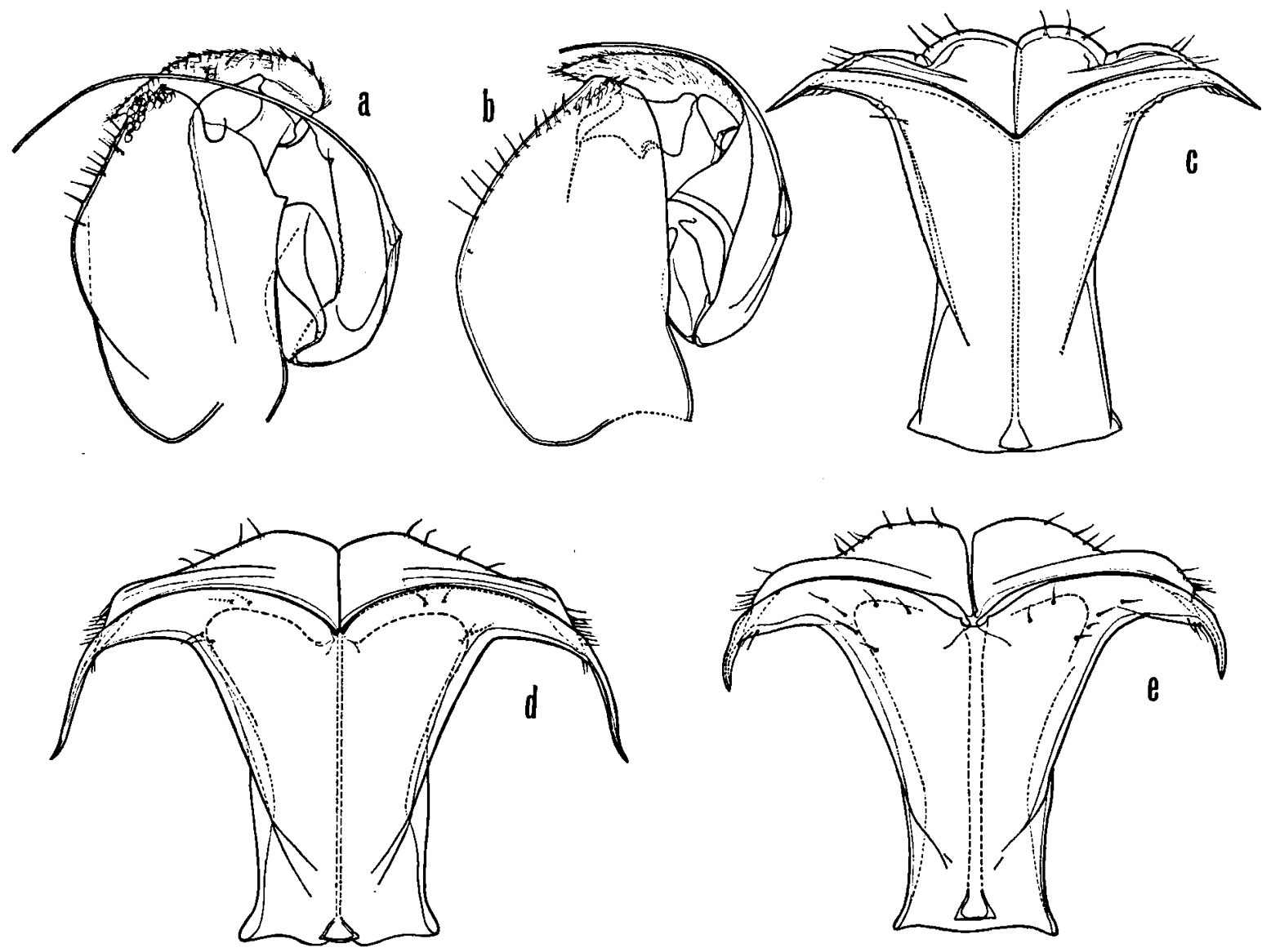

Fig. 6. a, Pléopode 2 de Jaera caspica; b, pléopode 2 de $J$. istri; c, préopercule de $J$. istri; d, préopercule de J. caspica; e, préopercule de $J$. sarsi. 
Le pléopode 2, qui porte le fouet copulateur, ressemble à celui des Jaera atlantiques et méditerranéennes bien que l'exopodite, bien dégagé du corps de l'appendice, s'étire en une lamelle pointue vers les marges externes (fig. $6 a-b$ ). Ces deux paires de pléopodes sont de même longueur que la chambre respiratoire et ont au repos une fonction operculaire.

L'organe cuticulaire de la femelle, comme chez les espèces de la Méditerranée, est une poche simple constituée d'une couche unique perdue et reconstituée à chaque cycle sexuel.

Il n'y a pas de dimorphisme sexuel au niveau des péréiopodes, normalement glabres. A peine observe-t-on l'apparition de quelques soies surnuméraires, courtes et droites, sur les trois paires de péréiopodes antérieurs des grands mâles de Jaera istri (fig. 8b).

Aucun appendice ne semble avoir de fonction spécifique lors de la précopulation, durant laquelle le mâle se maintient sur le dos de la femelle, souvent perpendiculairement au corps de celle-ci. La copulation se fait en position tête-bêche.

D'autres caractères morphologiques ont été signalés précédemment: l'élargissement du lobe du maxillipède, la garniture du corps constituée d'épines courtes et nombreuses, la chambre respiratoire cordiforme, surtout accusée chez $J$. istri, la présence de deux ongles sur tous les péréiopodes.

\section{Jaera istri $\mathrm{n}$. sp.}

Le pléotelson de cette espèce est court et ovale; il se différencie peu de celui de la femelle.

Les cornes du préopercule, assez courtes, pointent perpendiculairement à l'axe du corps (fig. $6 c$ ).

L'appendice proximal de l'endopodite du pléopode 2 possède un ergot en relief sur l'origine distale de la carène qui s'étire sur toute la longueur de l'article (fig. 6b).

La localité type de cette espèce est à Kladovo
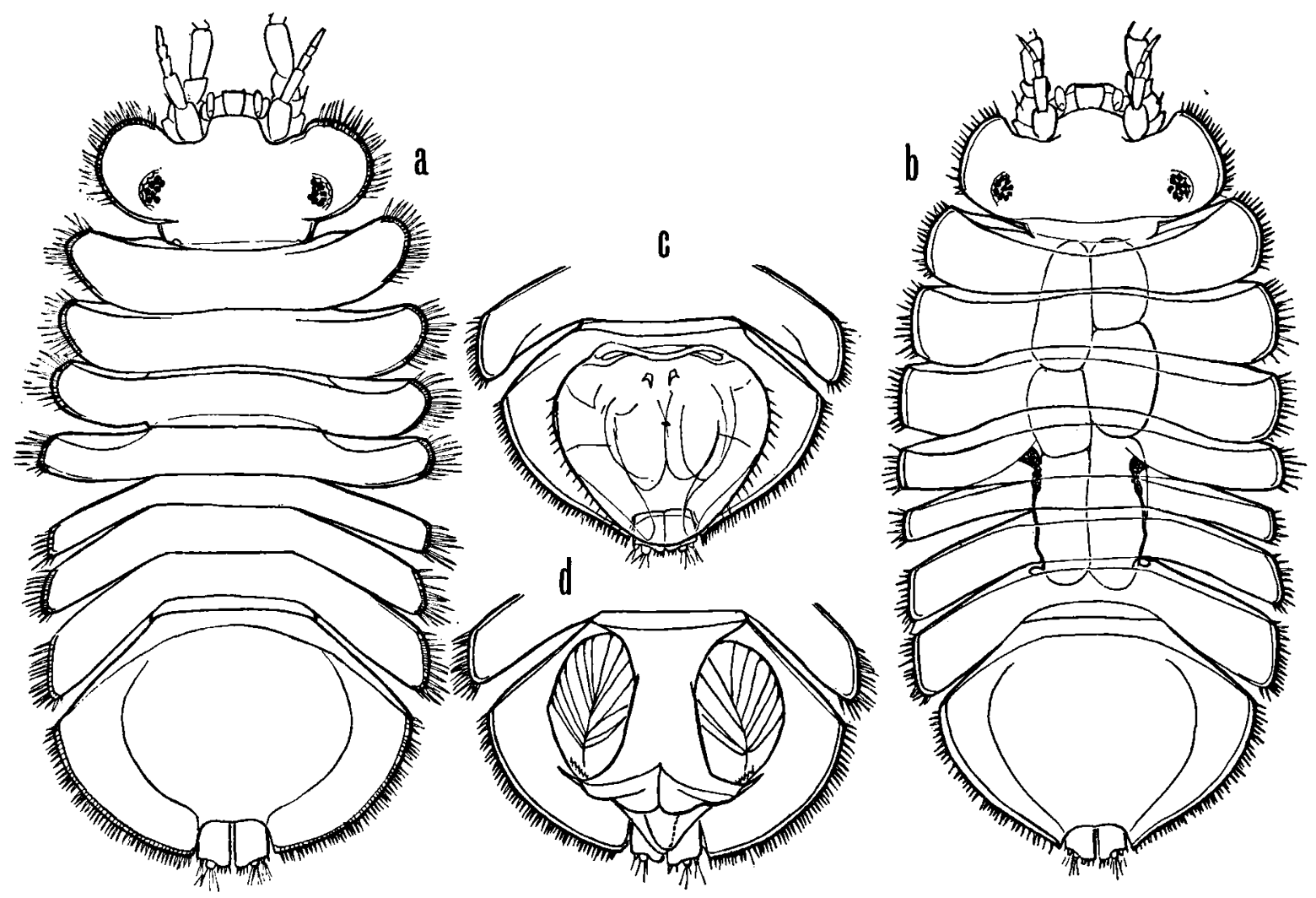

Fig. 7. a, Mâle de Jaera istri; b, femelle de J. istri; c, pléotelson de la femelle en vue ventrale; d, pléotelson du mâle en vue ventrale. 
en avant des Portes de Fer du Danube. Il est vraisemblable que les "Jaera sarsi" signalées dans ce fleuve par de nombreux auteurs appartiennent à cette espèce. Sa distribution irait alors de la Roumanie à la frontière austro-allemande.

Le type est un mâle de $1,98 \mathrm{~mm}$, déposé au $\mathrm{Mu}$ séum National d'Histoire Naturelle de Paris.

\section{Jaera sarsi Valkanov, 1938}

Le pléotelson mâle de Jaera sarsi est rendu quadrangulaire par son allongement longitudinal (fig. 8a). Le préopercule est de dimensions considérables avec des cornes plus longues que chez J. istri, parallèles à l'axe du corps et pointant vers l'avant (fig. 6e).

L'article proximal de l'endopodite du pléopode 2 est dépourvu de processus sclérifié.

Cette espèce vit en Bulgarie, où elle colonise les étendues saumâtres supralittorales qui bordent la Mer Noire. Valkanov (1938), le signale dans le Lac de Gebedze. Les exemplaires que je décris proviennent d'un échantillon du Lac de Schabla que m'a adressé le Dr. Abadieva.

\section{Jaera caspica Kesselyák, 1938}

= Jaera sarsi caspica Kesselyák, 1938. Cette espèce ressemble à Jaera istri, mais l'allongement du pléotelson est moins accusé et lui donne une forme subcirculaire (fig. 9a). Cette disposition semble également associée à la taille imposante du préopercule, avec de longues cornes dirigées vers l'avant du corps (fig. 6d).

Contrairement aux adultes, les immatures ne présentent pas de petites épines sur la bordure du corps, mais des soies de taille inégale, parfois très longues, qui disparaissent à la maturité sexuelle (fig. 9b).

Les exemplaires ayant servi à cette description proviennent de draguages effectués dans la Caspienne, à la hauteur de Tchecheng, par le Dr. Baguyrov.
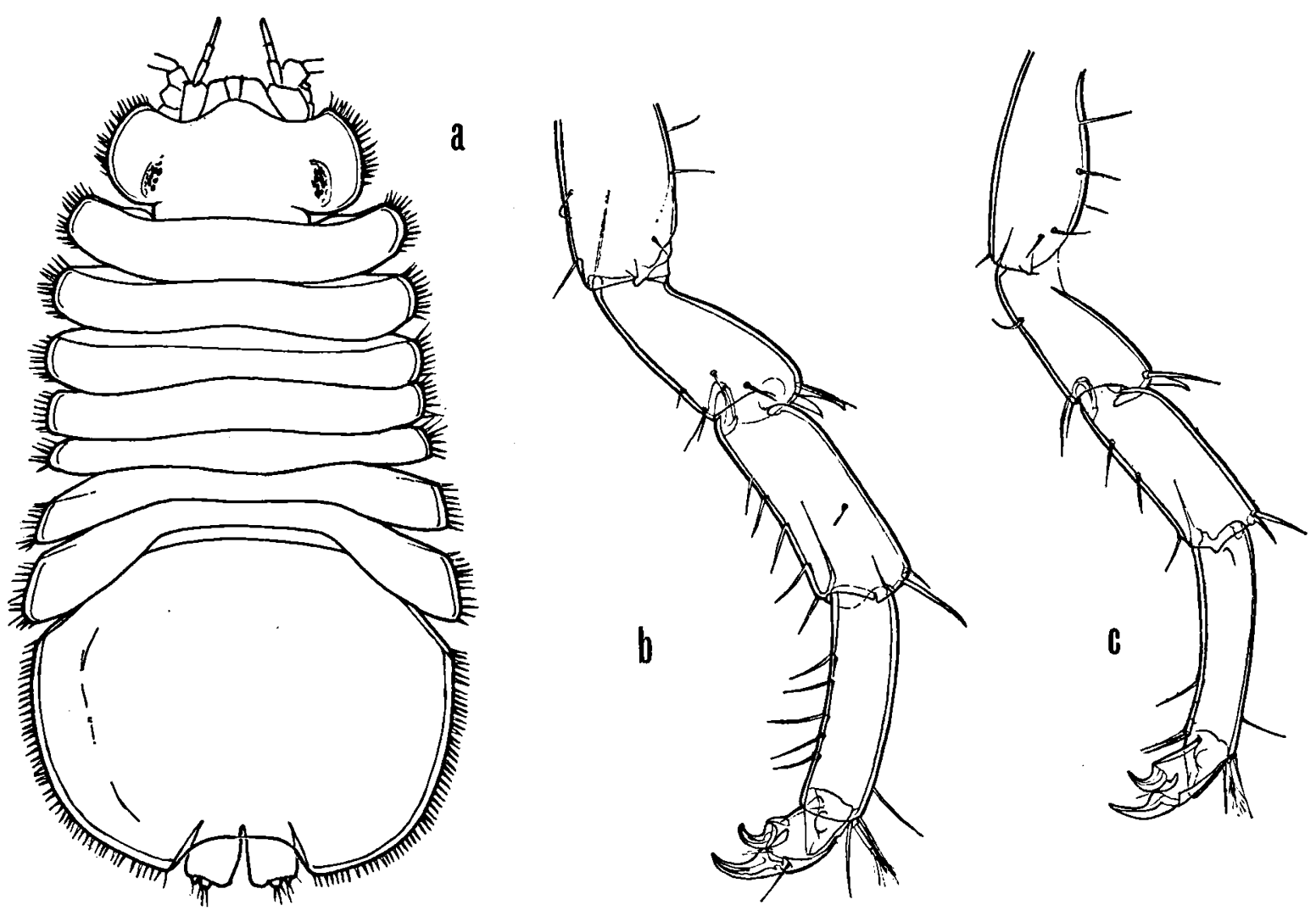

Fig. 8. a, Mâle de Jaera sarsi; b, péréiopode 2 mâle de J. istri; c, péréiopode 2 femelle de $J$. istri. 

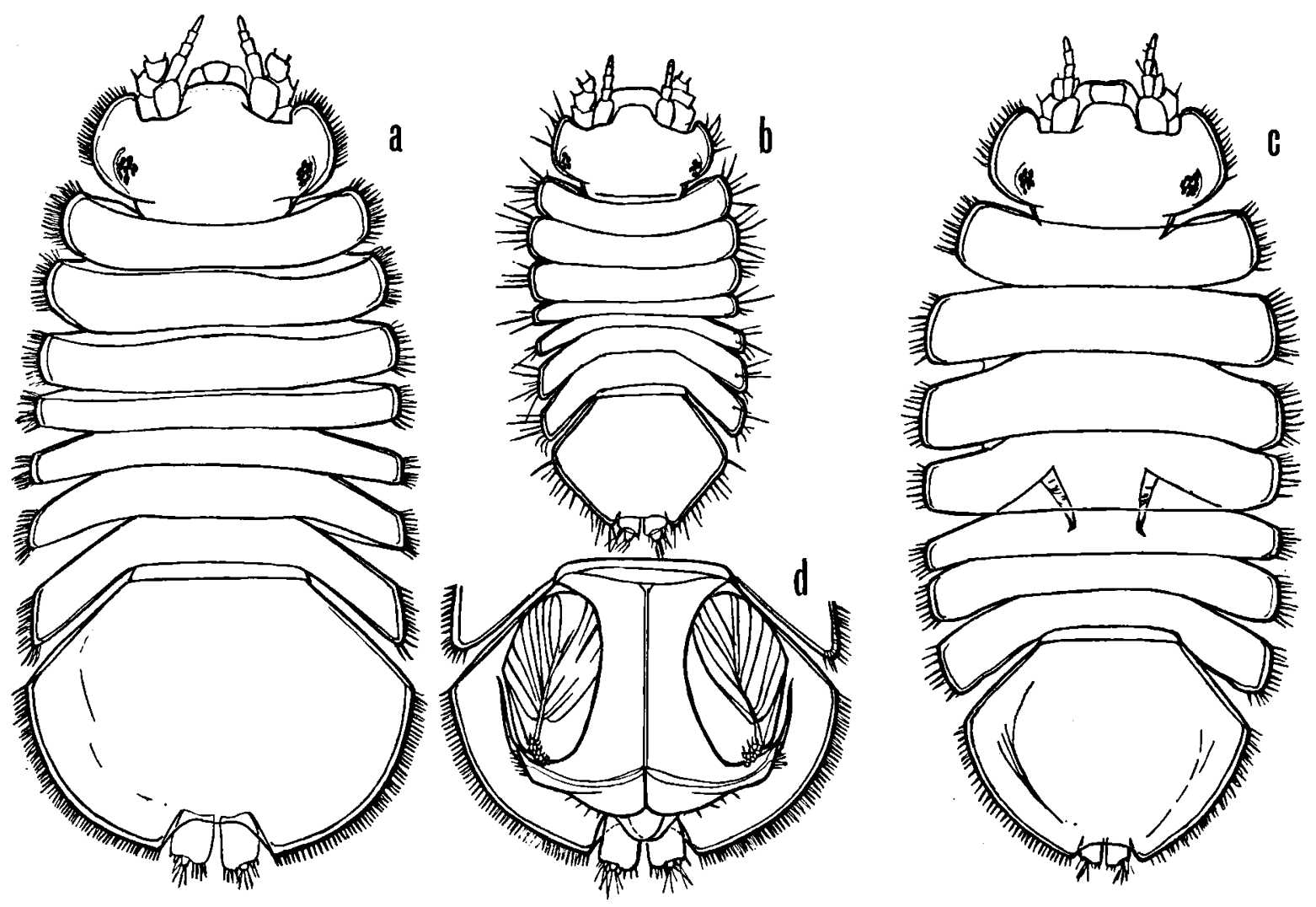

Fig. 9. a, Mâle de Jaera caspica; b, juvénile de la même espèce; c, femelle; d, pléotelson mâle en vue ventrale.

Remarques phylogéniques. - La découverte de ce groupe est liée à la description donnée par G. O. Sars (1897) d'une ,Jaera nordmanni" de Bakou, identifiée ultérieurement par la littérature comme une Jaera sarsi. En fait, G. O. Sars ne figure pas sur ses planches le préopercule qui seul permettrait de déterminer le spécimen, mais la représentation en vue dorsale qu'il donne de l'animal exclut son appartenance à l'espèce Jaera caspica, et en ferait plutôt une forme affine de Jaera istri. Il serait également intéressant de pouvoir donner une diagnose précise des ,Jaera sarsi"' signalées dans le Bug, le Dniepr, le Dniestr, le Don, la Volga et l'Oural par Birstein (1951) et Lyakhov \& Mordukhaĭ-Boltovskoĭ (1973). La difficulté de se procurer tout le matériel disponible de ce groupe fait que toute la richesse n'en est peut-être pas entièrement appréciée.

La présence de ce groupe dans les grands fleuves ponto-caspiens, dans la Caspienne et dans les lacs côtiers de la Mer Noire en font un groupe relicte typique de l'ancienne Paratéthys.

Les deux formes affines $J$. sarsi et $J$. caspica vivent en eau saumâtre, tandis que la forme danubienne $J$. istri est une espèce d'eau douce.

Ce groupe se distingue des autres Jaera par l'absence de dimorphisme sexuel secondaire. La taille et la forme du préopercule le rapprochent des espèces atlantiques, tandis que l'organe cuticulaire unistratifié, le cycle sexuel femelle et le pelstatisme l'apparentent au groupe méditerranéen.

\section{EVOLUTION DU GENRE JAERA}

La partie descriptive de cette révision a dégagé entre les quatre groupes naturels de Jaera des apparentements qui permettent d'en retrouver la phylogénie (fig. 10). J. hopeana, proche des lais et de la base des Jaera, se rapproche du groupe 


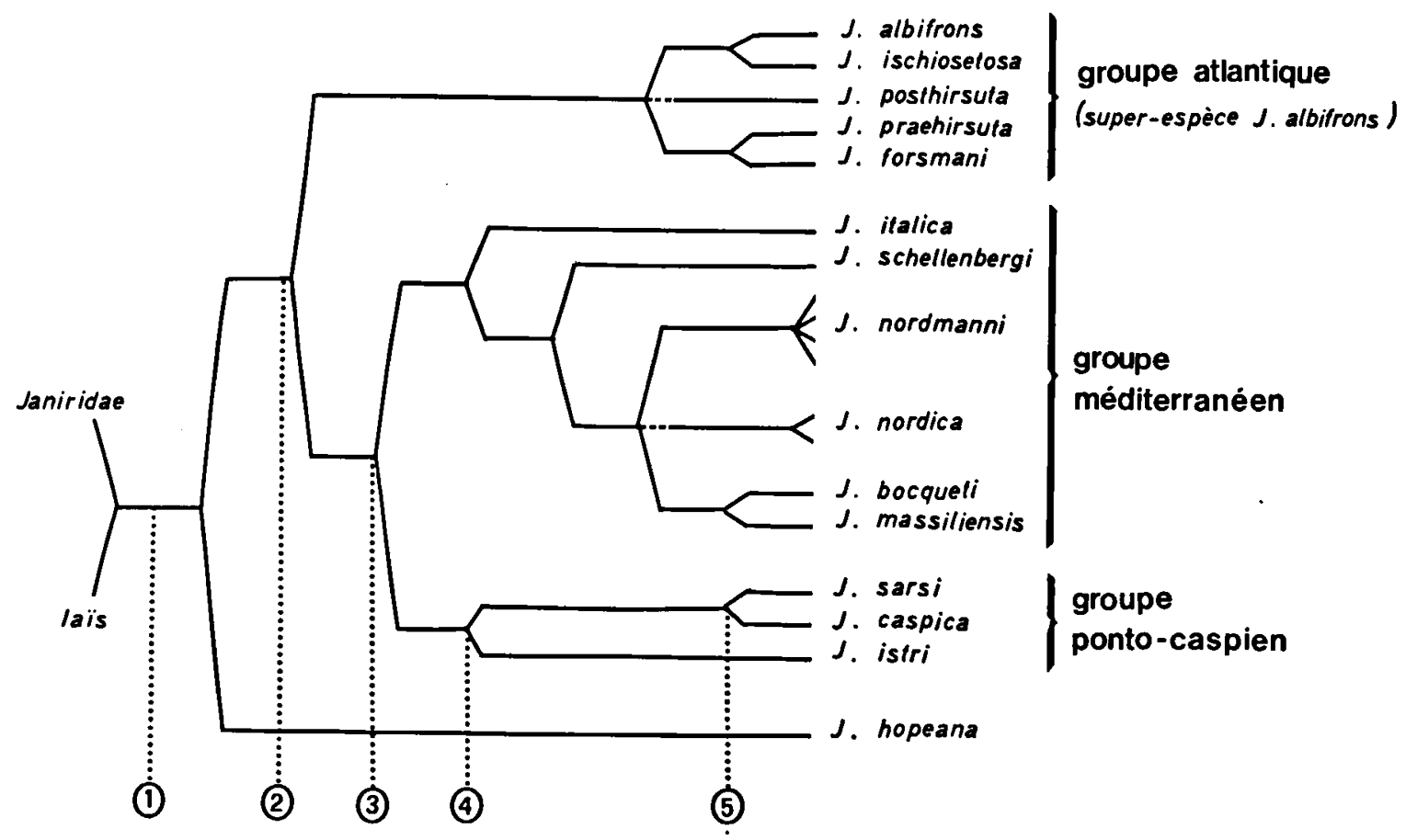

Fig. 10. Relations cladogénétiques vraisemblables entre les Jaera, selon la morphologie et la biologie sexuelle, et datations envisagées d'après la biogéographie. Les droites n'ont pas valeur de distance. 1, Isolement de la Méditerranée au Miocène (20 millions d'années A.P.); 2, isolement de la Paratéthys (15 M.A.); 3, formation du „Lago-mare” au Messinien (5,2 M.A.); 4, formation du lac des Carpathes (1,5 M.A.); 5, interglaciaire Riss-Würm. Les événements cladogénétiques situés à la droite du point 4 sont liés aux glaciations.

atlantique par sa biologie sexuelle femelle et le dimorphisme de la taille. Ce dernier constitue à son tour la charnière avec les deux autres groupes. De ceux-ci, c'est l'ensemble ponto-caspien qui rappelle le plus la super-espèce $]$. albifrons, par la morphologie de son préopercule, tandis qu'il s'apparente aux espèces méditerranéennes par l'organe cuticulaire unistratifié et le cycle sexuel de la femelle.

Le premier événement cladogénétique de l'évolution des Jaera a donc été l'individualisation de la lignée de $J$. hopeana, suivie de celle du groupe atlantique, qui se sépara alors de l'ancêtre commun des groupes méditerranéen et ponto-caspien, dont le dernier garde le plus de caractères primitifs.

$A$ de rares exceptions près, ce genre est typiquement européen et s'organise en groupes de parentés homogènes dont chacun occupe une province marine déterminée, avec peu de recouvrements. $\mathrm{Ce}$ parallélisme entre la classification des Jaera et l'agencement spatial des mers européennes met en évidence le rôle de celui-ci dans l'évolution du groupe: l'histoire de la spéciation des Jaera semble avoir été sous la dépendance des événements géologiques contemporains de leur radiation.

Liée à l'Océan Indien jusqu'au Miocène, la Téthys s'en sépare (20 millions d'années A.P.) et $s^{\prime}$ individualise en deux bras, la Méditerranée et la Paratéthys, communiquant entre elles et avec l'Atlantique (Hsü et al., 1977). Puis la Paratéthys, qui couvre l'Europe orientale, s'isole (15 M.A.) et devient un "lac-mer" aux eaux dessalées. Dans cette mer se constitue la faune saumâtricole „,pontocaspienne", qui se maintient jusqu'à nos jours malgré la courte transgression méditerranéenne du Messinien (5,2 M.A.). Au Pléistocène, la régression de la Paratéthys permet l'individualisation du lac des Carpathes (0,5 à 1,5 M.A.), vaste étendue d'eau douce correspondant à l'actuel bassin du Danube. La Caspienne devient un refuge pour la faune saumâtricole tandis que la Mer Noire forme un lac d'eau douce jusqu'au postglaciaire, où l'ou- 
verture du Bosphore le transforme en mer, isolant quelques espèces ponto-caspiennes dans les lacs et estuaires de sa côte occidentale.

$\mathrm{Au}$ cours de toute cette période, la Paratéthys reste coupée de la Méditerranée qui, après son isolement d'avec l'Atlantique (6 M.A.), subit une „crise de salinité" et un déssèchement total, sauf pendant quelques 100.000 ans, où le surcreusement du lit des fleuves yougoslaves causé par la profondeur du bassin asséché, draine une partie des eaux ponto-caspiennes et apporte dans le „Lago-Mare” quelques espèces de la Paratéthys.

L'ouverture du détroit de Gibraltar (5,2 M.A.) provoque la transgression de l'Atlantique dans les régions asséchées ou saumâtres de la Méditerranée qui deviendra, à dater du Pliocène, une mer de salinité normale.

L'histoire des Jaera semble suivre le cours de ces événements géologiques.

Lors de la jonction des continents africain et eurasiatique, qui isole la Méditerranée de l'Océan Indien, la lignée primitive des $J$. hopeana, exclusivement européennes, a pu se séparer des Iais de l'Indo-pacifique. C'est probablement à cette période que s'individualise le genre Jaera, et que s'effectue la séparation entre la condition sans doute ancestrale de ceux qui vivent sur les Sphaeromes ( $J$. bopeana), et la lignée des trois autres groupes, qui tout en restant littoraux, vivent librement à la face inférieure des pierres.

L'individualisation de la Paratéthys (15 M.A.) et l'assèchement de la Méditerranée scindent alors ces populations en deux lignées, selon qu'elles vivent dans les mers d'Europe orientale ou dans l'Atlantique. De ces dernières dérivent les Jaera albifrons. Les rivages de l'océan subissent peu de remaniements pendant la plus grande partie de cette période et offrent peu d'opportunités de spéciation. Mais au Pléistocène, les variations continuelles du niveau des mers et des lignes du rivage, le déplacement des zones climatiques tantôt brassent et tantôt séparent les populations: de là découle vraisemblablement la différenciation récente de ces animaux encore groupés en une „superespèce" aux formes interfécondes: ce qui s'est produit alors est illustré de nos jours par la distribution disjointe des $J$. posthirsuta américaines (fig. 11), repliées au sud lors de la dernière glaciation

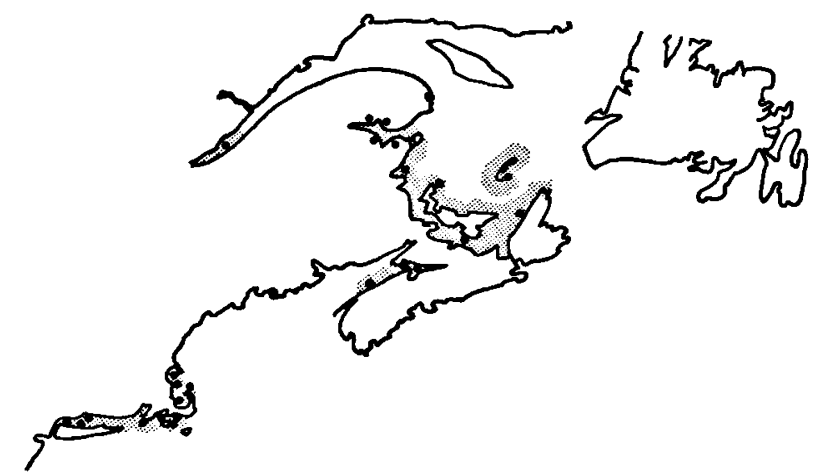

Fig. 11. Distribution de Jaera postbirsuta, montrant la disjonction postglaciaire de son aire de répartition en deux groupes de populations séparées par les eaux froides et exposées du nord-est des Etats Unis.

et dont certaines populations ayant remonté les côtes vers le nord lors de l'optimum climatique postglaciaire (6000 A.P.) se sont vues coupées du reste de l'espèce par la formation du courant du Labrador (Veuille, 1976).

La lignée parallèle qui poursuit son évolution dans la Paratéthys est l'ancêtre commun des groupes méditerranéen et ponto-caspien. Cela explique l'adaptation saumâtricole de toutes ces espèces, exceptées $J$. massiliensis et $J$. istri.

Le premier événement cladogénétique subi par le groupe ponto-caspien, et qui individualise l'espèce du Danube J. istri est apparemment lié à la formation du lac des Carpathes (1,5 M.A.); l'autre lignée donnera $J$. sarsi, de la côte Bulgare, et $J$. caspica, de la Caspienne. De nombreux auteurs, dont Zenkevitch (1957) et Mordukhai-Boltovskoi (1964) soulignent la fréquente parenté entre les couples d'espèces ou de sous-espèces qui vivent parallèlement dans la mer Caspienne et les lacs et estuaires relictuels des côtes de la Mer Noire. Il est tentant d'inférer de ces observations biogéographiques qu'à l'instar du reste de la faune ponto-caspienne, la spéciation de $J$. sarsi et $J$. caspica est d'origine glaciaire: l'ancêtre de ces deux formes, réfugié dans la Caspienne, aurait colonisé la Mer Noire à l'interglaciaire Riss-Würm, utilisant le détroit temporairement ouvert entre les deux mers par l'actuelle vallée de la Manytch (MordukhaiBoltovskoi, 1964). La fermeture de ce passage, puis la transgression méditerranéenne auraient isolé ces deux espèces dans leur disposition actuelle (fig. 12). 


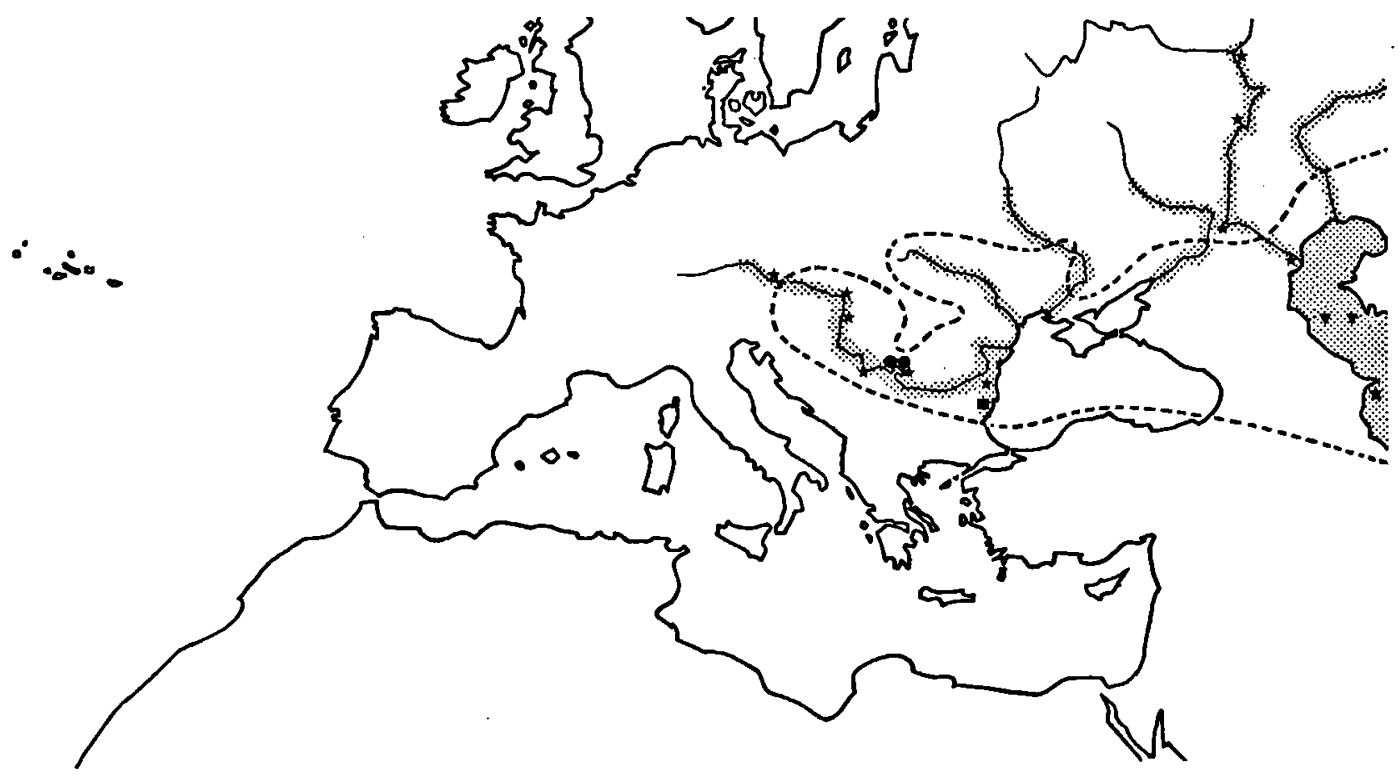

Fig. 12. Répartition des espèces ponto-caspiennes Jaera istri (cercles), J. sarsi (carrés) et J. caspica (triangles). Les populations de ce groupe d'espèces signalées avant leur révision systématique sont également indiquées (étoiles). Les limites approximatives de la Paratéthys sont tracées en tireté.

Les Jaera venues de la Paratéthys n'ont pu s'établir en Méditerranée que par la seule communication possible: celle du Messinien, où le "LagoMare" (5,2 M.A.) se constitue dans une partie de ce bassin, drainant les espèces des mers orientales qui donneront la faune relicte d'origine marine du relief karstique des Balkans. Les Jaera de cette lignée resteront un groupe principalement inféodé aux multiples petites stations d'eau saumâtre disséminées le long du rivage. Leur capacité de vivre en eau de mer (qu'atteste la possibilité d'élever des souches de laboratoire dans ce milieu) leur permet une bonne aptitude à la colonisation et au repeuplement de ces poches d'eau saumâtres d'existence souvent temporaire: c'est cette possibilité qui a permis à $J$. nordmanni et $J$. nordica de peupler les Açores, où elles remplacent la faune habituelle des Aselles et des Gammares dulçaquicoles (Monod, 1975), incapables de traverser l'immensité océanique qui sépare l'archipel volcanique du continent. L'importante diversification du groupe méditerranéen, qui voit se former dix espèces et sous-espèces en une période de cinq millions d'années, ne doit donc pas être recherchée dans des mécanismes d'isolement de nature écologique.

Les espèces se distribuent l'espace méditerranéen selon une zonation latitudinale (figs. 13, 14, 15). $J$. italica occupe le sud des bassins, J. nordmanni vit plus au nord et $J$. schellenbergi colonise le culde-sac septentrional de l'Adriatique. Si cette zonation a vraisemblablement été un caractère constant de ces espèces, sa disposition actuelle est forcément récente: J. nordmanni n'est présente en Mer Noire que depuis l'ouverture du Bosphore au cours du postglaciaire. Sa présence dans la zone centrale de l'Adriatique date de la même époque puisque la moitié nord de cette mer était émergée pendant la glaciation de Würm. Les trois groupes disjoints de J. nordmanni qui occupent la Méditerranée ont dû avoir autrefois une distribution plus méridionale. Avec les glaciations et le déplacement des zones climatiques, la zonation des Jaera a dû se déplacer vers le sud, permettant la jonction des populations des différents bassins. Le réchauffement du climat au postglaciaire, entraînant la remontée vers le nord des peuplements, a isolé les J. nordmanni des trois bassins, fermés au sud par l'expansion analogue des J. italica. De cette migration résulte l'éclatement géographique de cette espèce et le processus de sub-spéciation en cours. J. schellenbergi ne pouvait occuper pendant la glaciation würmienne que le sud de l'Ádriatique, 


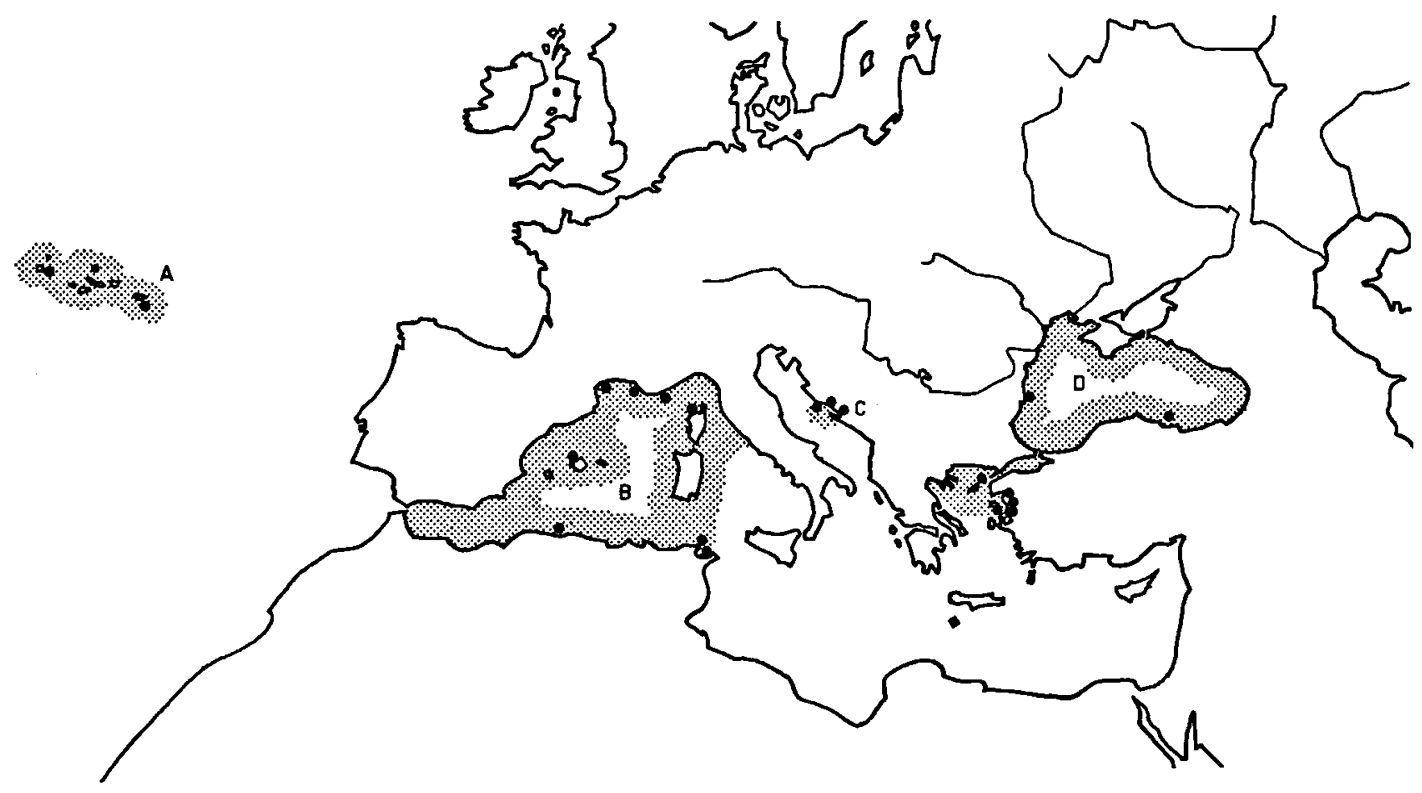

Fig. 13. Répartition disjointe des populations de Jaera nordmanni, montrant les quatre sous-espèces guernei (A), occidentalis (B), illyrica (C) et nordmanni (D).

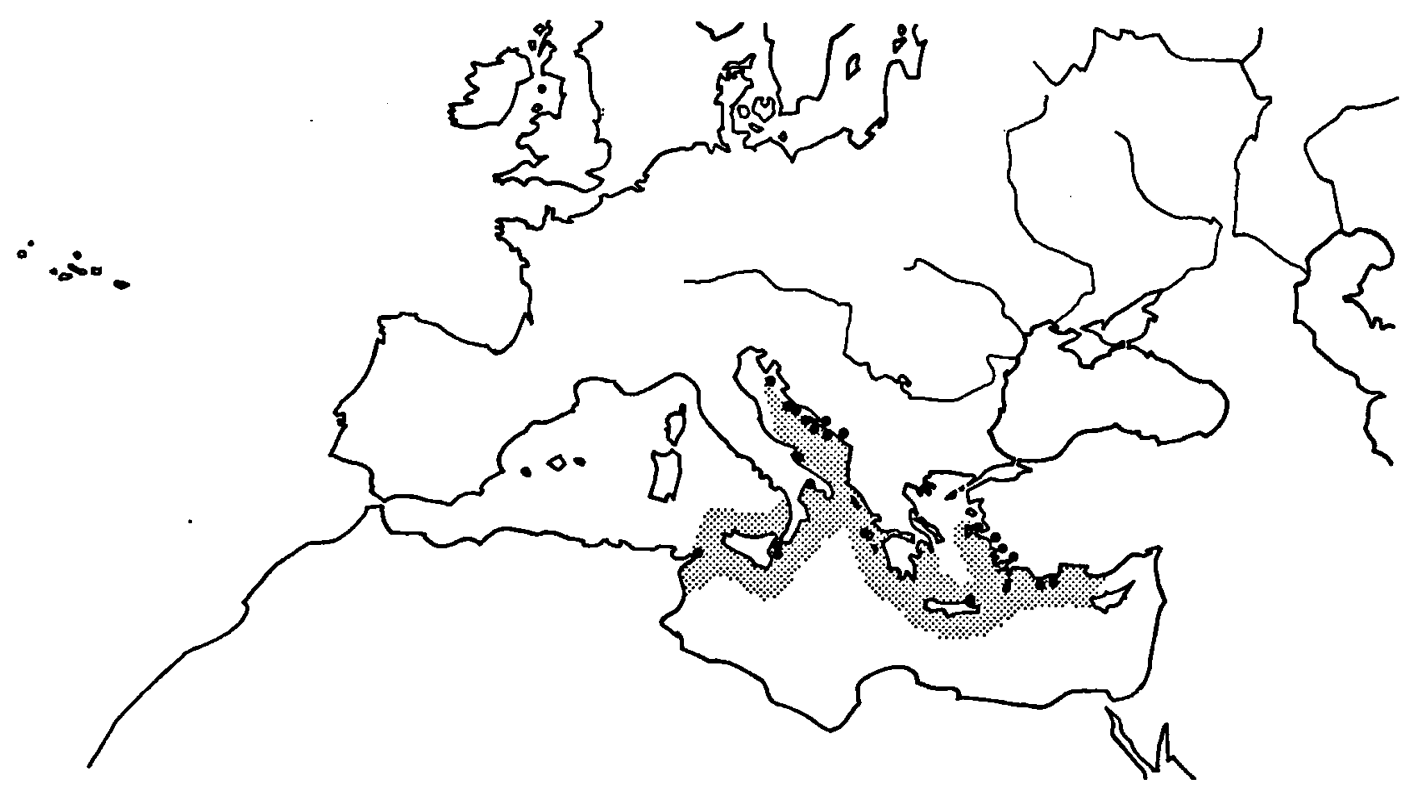

Fig. 14. Distribution de Jaera italica. 


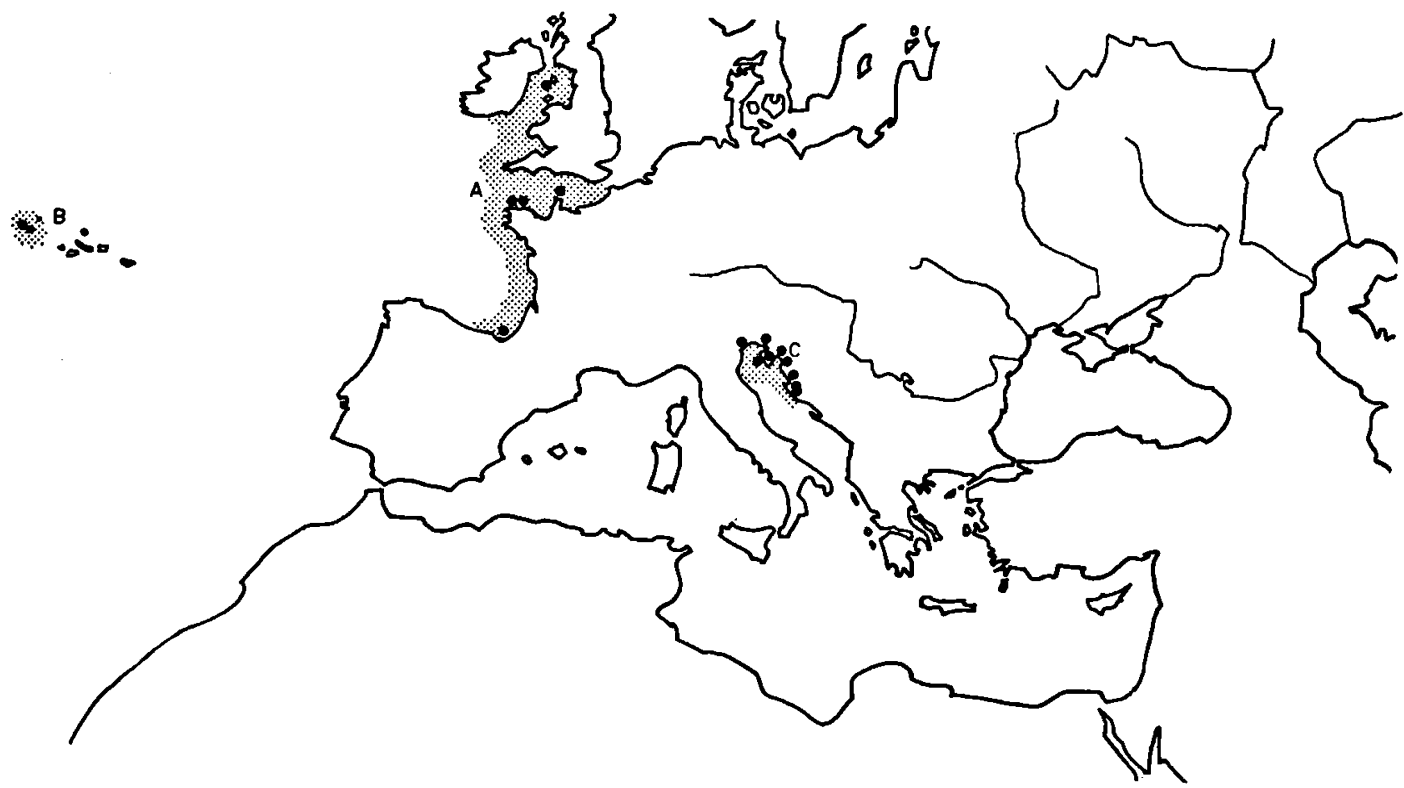

Fig. 15. Distribution de Jaera nordica nordica (A), J. nordica insulana (B) et J. schellenbergi (C).

à l'instar de l'espèce précédente, et a connu les mêmes migrations que cette espèce. Sa parenté étroite avec $J$. nordmanni amène l'hypothèse que sa spéciation résulte d'un même processus, advenu lors d'une glaciation antérieure. Il est même possible que les multiples situations d'allopatrie entretenues par les glaciations dans une mer aussi cloisonnée que l'est la Méditerranée soient entièrement responsables de la diversification des Jaera méditerranéennes.

Il résulte de l'ensemble de ces constatations que les faits géologiques actuellement connus peuvent rendre compte de façon satisfaisante de la spéciation allopatrique des espèces de Jaera: la formation des mers européennes, résultant des événements tectoniques de la fin du tertiaire, a entraîné une première diversification du genre. Les formes isolées dans chaque bassin ont été à l'origine des trois grands groupes actuels, que les glaciations ont à leur tour différenciés en de nombreuses espèces: l'ère glaciaire, loin d'avoir été la cause d'un appauvrissement de la faune préexistante, semble donc avoir été, dans ce cas précis, un puissant facteur d'évolution.

\section{REMERCIEMENTS}

Je remercie les Dr. Abadieva (Aquarium de Varna) et Bacesco (Muséum „G. Antipa” de Bucarest), pour les spécimens qu'ils m'ont fournis et les Dr. Piatakova, Baguyrov (Acadé. mie des Sciences d'Azerbaidjan) et Stevcic (Université de Zagreb, Station de Biologie de Roving) pour leur aide chaleureuse.

\section{BIBLIOGRAPHIE}

BACEsCo, M., 1948. Quelques observations sur la faune benthonique du défilé Roumain du Danube. Annls. scient. Univ. Jassy, 31 : 240-253.

Brrstein, J. A., 1951. Crustacea-Asellota. Fauna SSSR, 7 ( 5 ) 131-137.

Bocquet, C., 1953. Recherches sur le polymorphisme naturel des Jaera marina (Fabr.) (Isopodes Asellotes). Archs. Zool. exp. gén., 90: 187.450.

- 1972. La spéciation des Jaera albifrons. Etat présent des connaissances et des problèmes. In: B. BAtTAGLIA ed., Fifth European marine biology symposium: 131-138 (Piccin Editore, Padova).

Cariou, M. L., 1977. Recherches sur le polymorphisme enzymatique du complexe Jaera albifrons, Leach (Crustacé, Isopode): 1-76 (Thèse, Université de Paris VI).

ClÉRET, J. J., 1970. La pigmentation des Jaera albifrons Leach. V. Equilibre du système pigmentaire et gènes responsables du polychromatisme. Archs. Zool. exp. gén., 111: 447-493.

CoINEAU, N., 1971. Les Isopodes interstitiels: documents sur leur écologie et leur biologie. Mém. Mus. natn. Hist. nat. Paris, (N.S., A) 64: 1-170, pls. I-V.

CostA, A., 1853. Genere Jaera: Jaera Leach. In: O. G. CostA, Fauna del regno di Napoli, Crostacei-Isopodi, 83: 1.4 (Fr. Azzolino, Napoli).

Forsman, B., 1944. Beobachtungen über Jaera albifrons Leach an der Swedischen Westküste. Ark. Zool., 35A (11): 1.33 , pl. I.

- 1949. Weitere Studien über die Rassen von Jaera albifrons Leach. Zool. Bidr. Upps., 27: 451-463. 
Hennig, W., 1966. Phylogenetic systematics: 1-263 (University of Illinois Press, Urbana/Chicago/London).

Hsü, K. J., L. Montadert, D. Bernoulli, M. B. Citta, A. ERICKSON, R. E. Garrison, R. B. KiDD, F. MÈlierés, C. Müller \& R. WRIGHT, 1977. History of the Mediterranean salinity crisis. Nature, Lond., 267 (5610): 399-403.

HuRLEY, D. E., 1956. The New Zealand species of Iais (Crustacea Isopoda). Trans. R. Soc. N.Z., 83: 715-719.

JAżDżEWSKI, K., 1969. Ecology and biology of species of the Jaera albifrons group (Isopoda Asellota) in the bay of Puck, Polish Baltic Sea. Crustaceana, 17: 266-281.

JoNES, M. B., 1972a. Osmoregulation in the Jaera albifrons group of species (Isopoda, Asellota). J. mar. biol. Ass. U.K., 52 (2): 419-427.

— 1972b. The mouthparts of the members of the Jaera albifrons group of species (Crustacea, Isopoda). Mar. Biol. Berlin, 11: 264-270.

JoNes, M. B. \& E. NAYLoR, 1971. Breeding and bionomics of the British members of the Jaera albifrons group of species (Isopoda: Asellota). J. Zool., Lond., 165 (2): 183-199.

Karaman, S., 1953. Über die Jaera-Arten Jugoslaviens. Acta adriat., 5 (5): 1-20.

Kesselyák, A., 1938. Die Arten der Gattung Jaera Leach (Isopoda Asellota). Zool. Jb. (Syst. Ökol. Geogr. Tiere), 71: 219-252.

Kotré, P., 1968. Hypania invalida (Polychaeta sedentaria) und Jaera sarsi (Isopoda) erstmals in der deutschen Donau. Arch. Hydrobiol., Suppl. 34 (1-2): 88-114.

LEACH, W. E., 1814 (1813). Crustaceology. In: D. BREWSTER ed., The Edinburgh encyclopaedia, 7: 383-437 (W. Blackwood, Edinburgh).

LÉCHER, P., 1964. Recherches complémentaires sur le polytypisme de la super-espèce Jaera albifrons Leach = Jaera marina Fabricius. III. Etude chromosomique de différentes populations de Jaera (albifrons) syei Bocquet. Bull. biol. Fr. Belg., 98: 415-431.

- 1967. Cytogénétique de l'hybridation expérimentale et naturelle chez l'Isopode Jaera (albifrons) syei Bocquet. Archs. Zool. exp. gén., 108: 633-698, pls. I-VI.

LEMERCIER, A., 1955. Caractères morphologiques des pièces buccales de Jaera marina (Fabr.). Mém. Soc. natn. Sci. nat. Math. Cherbourg, (5) 47 (7): 86-95.

- 1960 . La super-espèce Jaera nordmanni (Rathke) (Isopodes, Asellotes, Janiridae). Crustaceana, 1 (1): 9-27.

- 1968. Isopoda Asellota: Janiridae from the Azores. Bolm. Mus. munic. Funchal, 22 (99): 30-31.

LyAKHOV, S. M. \& F. D. MORDUKhai-BoltovsKoI, 1973. O rasprostranenii Jaera sarsi Valkanov, 1936 (Crustacea, Isopoda) v basseïne Volgi. Inf. Byul. Biol. Vnutr. Vod., 20: $29-33$.

Menzies, R. J. \& J. L. Barnard, 1951. The isopodan genus Iais (Crustacea). Bull. Sth. Calif. Acad. Sci., 50 (3): 136-151, pls. I-IX.

MONOD, TH., 1925. Tanaidacés et Isopodes aquatiques de l'Afrique occidentale et septentrionale, 2e partie: Tanaidacea (fin), Anthuridae (fin), Stenetriidae, Jaeridae (Janirini), Munnidae (Munnini), Asellidae, Limnoriidae. Bull. Soc. Sci. nat. Maroc, 5 (3): 233-247, pls. XLII-LII.

—, 1975. Sur la distribution de quelques Crustacés Malacostracés d'eau douce ou saumâtre. XVIIe Congr. int. Zool., Monaco, 25-30 Sep. 1972. In: Mém. Mus. natn. Hist. Nat. Paris, (A) (Zool.) 88: 98-105.
Mordukhal-Boltovskor, PH. D., 1964. Caspian fauna beyond the Caspian sea. Int. Revue ges. Hydrobiol., 49 (1): $139-176$

NAYLOR, E., 1972. British marine isopods. Keys and notes for the identification of the species. Synopses Br. Fauna, (N.S.) 3: 1.86 (published for the Linnean Society of London by Academic Press, London/New York).

NAYLOR, E. \& I. HAAHTElA, 1966. Habitat preference and interspersion of species within the superspecies Jaera albifrons Leach (Crustacea: Isopoda). J. anim. Ecol., 35: 209-216.

— \& - 1967. Quantitative ecological distribution of the Jaera albifrons group of species in the Baltic. Ophelia, 4: $19-27$.

NAYLOR, E. \& D. J. SLINN, 1958. Observations on the ecology of some brackish water organisms in pools at Scarlett Point, Isle of Man. J. anim. Ecol., 27: 15-25.

Prunus, G., 1968. Etude de systématique des populations chez l'Isopode Jaera (albifrons) albifrons Forsman. Archs. Zool. exp. gén., 109: 643-702.

Prunus, G. \& G. Pantoustier, 1976. Le genre Jaera Leach (Isopode Asellote) sur les côtes de Tunisie. Etude morphologique et écologique. Archs. Zool. exp. gén., 117: 235-254.

RAThKe, H., 1837. Beitrag zur Fauna der Krym. Mém. prés. Acad. Imp. Sci. St. Petersb., 3: 291-454, pls. I-X.

Rossi, C., 1906. Sulla convivenza di due isopodi del Mediterraneo. Zool. Anz., 30: 107-109.

Rotramel, G. L., 1975. Observations on the commensal relations of Iais californica (Richardson, 1904) and Sphaeroma quoyanum $\mathrm{H}$. Milne Edwards, 1840 (Isopoda). Crustaceana, 28 (3): 247.256.

SARS, G. O., 1897. On some additional Crustacea from the Caspian Sea. Ezheg. zool. Muz., 2 (3): 273-305, pls. XIII-XVI.

SchmalfUSs, H., 1975. Neues Isopoden-Material aus Griechenland. Sber. öst. Akad. Wiss., Math.-naturw. Kl., (Abt. I) 184 (1-5): 27-66.

SJöBERG, B., 1967. On the ecology of the Jaera albifrons group (Isopoda). Sarsia, 29: 321-348.

, 1969. Population studies of the Jaera albifrons group. Limnologica, Berlin, 7: 69-73.

- , 1970. Population density, size, age, reproduction and microdistribution in the Jaera albifrons group (Isopoda). Oikos, 21: 241-247.

SkET, B., 1969. UUber die Verbreitung einiger Malacostraca (Hadzia, Salentinella, Nengammarus, Jaera) langs der Jugoslavischen Adria-Küste. Bull. scient. Cons. Acad. RSF Yougosl., (A) 14 (5-6): 147-148.

SOKal, R. R. \& P. H. A. SNEATH, 1963. Principles of numerical taxonomy: i-xvi, 1-359 (W. H. Freeman, San Francisco/London).

Staiger, H. \& C. Boceuet, 1954. Cytological demonstration of female heterogamety in isopods. Experientia, 10 (2): 64-66.

__ \& $\$$ - 1956. Les chromosomes de la super-espèce Jaera marina (F.) et de quelques autres Janiridae (Isopodes Asellotes). Bull. biol., 90 (1): 1-32.

Steele, D. J. \& V. J. Steele, 1972. The biology of Jaera spp. (Crustacea, Isopoda) in the north-western Atlantic. 1. Jaera ischiosetosa. Can. J. Zool., 50: 205-211.

Strouhal, H., 1942. Vorläufige Mitteilung über die von $M$. Beier in Nordgriechenland gesammelten Asseln. Zool. Anz., 138: 145-162.

VAlKanov, A., 1938. Uebersicht der europäischen Vertretern 
der Gattung Jaera Leach 1813 (Isopoda Genuina). God. sof. Univ. Sofiya Fiz.-Mat. Fak., 34 (3, Sci. nat.): 53-78.

Verhoeff, K. W., 1943. Zur Morphologie, Ökologie und Systematik von Sphaeroma, Europosphaera und Jaera. Z. Morph. Okol. Tiere, 40 (2): 276-290.

, 1949. Zur Kenntnis der maritimen Isopoden-Gattung Sphaeroma, die Incurvation derselben und Jaera als Gast von Sphaeroma. 81. Isopoden-Aufsatz. Arch. Hydrobiol., 42: $395.422 \mathrm{~b}$.

Veuille, M., 1976. Biogeography of the Jaera albifrons superspecies (Isopoda, Asellota) on the Atlantic coast of Canada. Can. J. Zool., 54 (8): 1235-1244.

_, 1977. Systématique et affinités continentales des Jaera (Isopodes, Asellotes) de l'île de Florès (Açores). Bull.
Mus. natn. Hist. nat. Paris, (3) 1976 (415) (Zool., 292): 1327-1338.

1978a. Biologie de la reproduction chez Jaera (Isopode Asellote). I. Structure et fonctionnement des pièces copulatrices mâles. Cah. Biol. mar., 19 (3): 299-308, pl. I. 1978b. Biologie de la reproduction chez Jaera (Isopode Asellote). II. Evolution des organes reproducteurs femelles. Cah. Biol. mar., 19 (4): 385-395, pl. I.

Veuille, M. \& A. Kocatas, sous presse. Les Jaera (Isopodes, Asellotes) des côtes de Turquie: Etude comparative et biogéographique. Vie Milieu.

Zenkevitch, L. A., 1957. Caspian and Aral Seas. In: J. W. HeDGPETH ed., Treatise on marine ecology and paleoecology. Mem. geol. Soc. Am., 67 (1): 891-916. 